\title{
Vibration analysis of a mass on a spring by means of magnus expansion method
}

\author{
Musa Basbuk ${ }^{1}$, Aytekin Eryilmaz ${ }^{1}$ and Mehmet Tarik Atay ${ }^{2}$ \\ ${ }^{1}$ Department of Mathematics, Nevsehir Haci Bektas Veli University, Nevsehir, Turkey \\ ${ }^{2}$ Department of Mechanical Engineering, Abdullah Gul University, Kayseri, Turkey
}

Received: 15 August 2015, Revised: 10 September 2015, Accepted: 16 September 2015

Published online: 10 April 2016

\begin{abstract}
In this paper, the differential equation for the motion of a mass on a spring is investigated, solutions of six different cases were analyzed and numerical solutions are obtained by means of Magnus Expansion Method. Any truncation of the Magnus series preserves qualitative geometric properties of the exact solution (Castellano et al. 2014). This is an important advantage of the Magnus expansion method. Therefore Magnus expansion method provides more accurate solutions than other frequently used numerical schemes. Finally the numerical results obtained by the present method and the analytical results were compared.
\end{abstract}

Keywords: Magnus Expansion, Vibration, Free Damped Motion, Free Undamped Motion, Forced Motion.

\section{Introduction}

In 1954, Magnus provided an exponential representation of the solution of a first order linear homogeneous differential equation for a linear operator that named Magnus Expansion. Since 1960's the Magnus expansion has been successfully applied in various areas of physics and chemistry, from atomic and molecular physics to nuclear magnetic resonance and quantum electrodynamics. Magnus neither derived a general formula for Magnus expansion nor proved conditions for convergence. Several authors such as Bialynicki-Birula, Mielnik \& Plebanski (1969), Mielnik \& Plebanski (1970), Strichartz (1987) and Fomenko \& Chakon (1990) presented a formula for general terms in the Magnus expansion but they are too complicated and not practical for using at high orders. Iserles \& Norsett (1999) presented a practical recursive algorithm that generates the terms of Magnus expansion. In the study of linear ordinary differential equations arises an infinite series which is called Magnus series. If the series converges, then the matrix exponential of the sum equals the fundamental solution of the differential equation. Blanes et al. (1998) considered the approximate solutions of matrix linear differential equations by matrix exponentials and the convergence issue of Magnus and Fer expansions. They obtained the upper bounds for the convergence radius in terms of the norm of the defining matrix of the system. Moan \& Niesen (2008) considered the question: When does the series converge? The main result they obtained, established a necessary condition for convergence. The first physical application of Magnus Expansion is Robinson's (1963) work. Since then, the Magnus Expansion became rapidly popular. It has been used in quantum mechanics to study time-dependent problems (Pechukas \& Light 1966), semiclassical atomic collisions theory (Baye \& Heenen 1973), the behaviour of molecular systems in intense laser fields (Milfeld \& Wyatt 1983), multiphoton excitation of molecules (Schek, Jortner \& Sage 1981), pulsed magnetic resonance spectra (Evans 1968), spectral line broadening (Cady 1974), infrared divergences in QED (Dahmen, Scholz \& Steiner 1982), the solar neutrino problem (MSW effect) (D’Olivo \& Oteo 1990) and a trajectories solution of the Hamilton equations in classical mechanics (Oteo \& Ros 1991), transition amplitude and the cross section for K-shell ionization of atoms by heavy-ion impact (Eichler 1977), the time-evolution of rotationally induced inner-shell excitation in atomic collisions (Wille 1981; Wille \& Hippler 1986), the theoretical study 
of electron-atom collisions, involving many channels coupled by strong, long-range forces (Hyman 1985), the theory of the pressure broadening of rotational spectra (Cady 1974), computing propagation in optical waveguides (Lu 2006), Helmholtz equation in waveguides (Lu 2005; 2007), non-holonomic motion planning of systems without drift (Duleba 1997; 1998), among non-holonomic systems there are free-floating robots, mobile robots and underwater vehicles (Murray, Li \& Satry 1994). Also new ideas emerged for the algorithm used as an efficient numerical integrator (Iserles \& Nørsett 1997; 1999). As an application of the Magnus Expansion Method, the vibration analysis of a mass on a spring is considered.

Vibration is a repetitive, periodic, or oscillatory response of a mechanical system (De Silva, 2000). Problems involving vibration occur in many areas of mechanical, electronics, geological, civil and aerospace engineering. In general vibration is undesirable since it wastes energy, makes unwanted sound and noise and reduces efficiency and may be harmful or dangerous. In this study we apply the magnus series method to the differential equations that occur in the vibration of a mass on a spring. And we compare the results obtained by the present method with the exact solutions are given in (Ross 1984).

Our paper is organized as follows. In Section 2 the Magnus series method of order 4 and 6 for linear ordinary differential equations is investigated. In Section 3 the vibration analysis of a mass on a spring is introduced. Some numerical experiments with six different cases are performed in Section 4.

\section{Magnus expansion}

The linear differential equation on a matrix Lie-group is an equation of the form

$$
Y^{\prime}=A(t) Y, \quad t \geq 0, \quad Y(0)=Y_{0} \in G
$$

where $A: \rightarrow$ is the matrix function, is the Lie group, is the Lie algebra of the corresponding Lie-group. Magnus (1954) expressed the solution of equation (1) as the exponential of a certain function,

$$
Y(t)=e^{\Omega(t)}
$$

Theorem 1. (Magnus 1954) Let $A(t)$ be a known function of $t$ (in general, in an associative ring), and let $Y(t)$ be an unknown function satisfying (1) with $Y(0)=I$. Then, if certain unspecified conditions of convergence are satisfied, $Y(t)$ can be written in the form

$$
Y(t)=\exp (\Omega(t))
$$

where

$$
\frac{d \Omega}{d t}=\sum_{n=0}^{\infty} \frac{B_{n}}{n !} \operatorname{ad}_{\Omega}^{n} A,
$$

and $B_{n}$ are the Bernoulli numbers. Integration of (4) by iteration leads to an infinite series for the first terms of which are

$$
\Omega(t)=\int_{0}^{t} A\left(t_{1}\right) d t_{1}-\frac{1}{2} \int_{0}^{t}\left[\int_{0}^{t_{2}} A\left(t_{1}\right) d t_{1}, A\left(t_{2}\right)\right] d t_{2}+\cdots
$$

For the proof see Blanes et al. (2009).

Magnus (1954) obtained an infinite recursive series for $\Omega$ by solving the equation (4) with Picard's iteration as follows

$$
\Omega_{0} \equiv 0,
$$




$$
\Omega_{n+1}=\int_{0}^{t} \operatorname{dexp}_{\Omega_{n}}^{-1} A(\xi) \mathrm{d} \xi=\sum_{k=0}^{\infty} \frac{B_{k}}{k !} \int_{0}^{t} \operatorname{ad}_{\Omega_{n}}^{k} A(\xi) \mathrm{d} \xi, \quad n=0,1,2, \ldots
$$

Substituting the equation (6) into the equation (7) one can get the $\Omega_{i}$ for $i=1,2,3, \ldots$ respectively

$$
\begin{aligned}
& \Omega_{1}=\int_{0}^{t} A\left(t_{1}\right) d t_{1} \\
& \Omega_{2}=\int_{0}^{t} A\left(t_{1}\right) d t_{1}-\frac{1}{2} \int_{0}^{t}\left[\int_{0}^{t_{2}} A\left(t_{1}\right) d t_{1}, A\left(t_{2}\right)\right] d t_{2}+\cdots \\
& \Omega_{3}=\int_{0}^{t} A\left(t_{1}\right) d t_{1}-\frac{1}{2} \int_{0}^{t}\left[\iint_{0}^{t_{2}} A\left(t_{1}\right) d t_{1}, A\left(t_{2}\right)\right] d t_{2}+\frac{1}{12} \int_{0}^{t}\left[\int_{0}^{t_{3}} A\left(t_{2}\right) d t_{2},\left[\int_{0}^{t_{3}} A\left(t_{2}\right) d t_{2}, A\left(t_{3}\right)\right]\right] d t_{3} \\
& +\frac{1}{4} \int_{0}^{t}\left[\int_{0}^{t_{3}}\left[\int_{0}^{t_{2}} A\left(t_{1}\right) d t_{1}, A\left(t_{2}\right)\right] d t_{2}, A\left(t_{3}\right)\right] d t_{3}+\cdots \\
& \Omega_{4}=\int_{0}^{t} A\left(t_{1}\right) d t_{1}-\frac{1}{2} \int_{0}^{t}\left[\iint_{0}^{t_{2}} A\left(t_{1}\right) d t_{1}, A\left(t_{2}\right)\right] d t_{2}+\frac{1}{12} \int_{0}^{t}\left[\int_{0}^{t_{3}} A\left(t_{2}\right) d t_{2},\left[\int_{0}^{t_{3}} A\left(t_{2}\right) d t_{2}, A\left(t_{3}\right)\right]\right] d t_{3} \\
& +\frac{1}{4} \int_{0}^{t}\left[\iint_{0}^{t_{3}}\left[\int_{0}^{t_{2}} A\left(t_{1}\right) d t_{1}, A\left(t_{2}\right)\right] d t_{2}, A\left(t_{3}\right)\right] d t_{3}-\frac{1}{24} \int_{0}^{t}\left[\int_{0}^{t_{4}} A\left(t_{3}\right) d t_{3},\left[\int_{0}^{t_{4}}\left[\int_{0}^{t_{3}} A\left(t_{2}\right) d t_{2}, A\left(t_{3}\right)\right] d t_{3}, A\left(t_{4}\right)\right]\right] d t_{4} \\
& -\frac{1}{24} \int_{0}^{t}\left[\int_{0}^{t_{4}}\left[\int_{0}^{t_{3}} A\left(t_{2}\right) d t_{2}, A\left(t_{3}\right)\right] d t_{3},\left[\int_{0}^{t_{4}} A\left(t_{3}\right) d t_{3}, A\left(t_{4}\right)\right]\right] d t_{4}-\frac{1}{24} \int_{0}^{t}\left[\iint_{0}^{t_{4}}\left[\int_{0}^{t_{3}} A\left(t_{2}\right) d t_{2},\left[\int_{0}^{t_{3}} A\left(t_{2}\right) d t_{2}, A\left(t_{3}\right)\right]\right] d t_{3}, A\left(t_{4}\right)\right] d t_{4} \\
& -\frac{1}{8} \int_{0}^{t}\left[\iint_{0}^{t_{4}}\left[\int_{0}^{t_{3}}\left[\int_{0}^{t_{2}} A\left(t_{1}\right) d t_{1}, A\left(t_{2}\right)\right] d t_{2}, A\left(t_{3}\right)\right] d t_{3}, A\left(t_{4}\right)\right] d t_{4}+\cdots
\end{aligned}
$$

The Magnus series expansion is,

$$
\Omega(t)=\sum_{k=0}^{\infty} H_{k}(t)
$$

where each $H_{k}$ includes exactly $k+1$ integrals and $k$ commutators (Iserles et al. 2000). Thus,

$$
\begin{aligned}
& H_{0}(t)=\int_{0}^{t} A\left(t_{1}\right) d t_{1} \\
& H_{1}(t)=-\frac{1}{2} \int_{0}^{t}\left[\int_{0}^{t_{2}} A\left(t_{1}\right) d t_{1}, A\left(t_{2}\right)\right] d t_{2} \\
& H_{2}(t)=\frac{1}{12} \int_{0}^{t}\left[\int_{0}^{t_{3}} A\left(t_{2}\right) d t_{2},\left[\int_{0}^{t_{3}} A\left(t_{2}\right) d t_{2}, A\left(t_{3}\right)\right]\right] d t_{3}+\frac{1}{4} \int_{0}^{t}\left[\int_{0}^{t}\left[\int_{0}^{t_{2}} A\left(t_{1}\right) d t_{1}, A\left(t_{2}\right)\right] d t_{2}, A\left(t_{3}\right)\right] d t_{3} \\
& H_{3}(t)=-\frac{1}{24} \int_{0}^{t}\left[\int_{0}^{t_{4}} A\left(t_{3}\right) d t_{3},\left[\int_{0}^{t_{4}}\left[\int_{0}^{t_{3}} A\left(t_{2}\right) d t_{2}, A\left(t_{3}\right)\right] d t_{3}, A\left(t_{4}\right)\right]\right] d t_{4}-\frac{1}{24} \int_{0}^{t}\left[\int_{0}^{t_{4}}\left[\int_{0}^{t_{3}} A\left(t_{2}\right) d t_{2}, A\left(t_{3}\right)\right] d t_{3},\left[\int_{0}^{t_{4}} A\left(t_{3}\right) d t_{3}, A\left(t_{4}\right)\right]\right] d t_{4} \\
& -\frac{1}{24} \int_{0}^{t_{0}}\left[\int_{0}^{t_{4}}\left[\int_{0}^{t_{3}} A\left(t_{2}\right) d t_{2},\left[\int_{0}^{t_{3}} A\left(t_{2}\right) d t_{2}, A\left(t_{3}\right)\right]\right] d t_{3}, A\left(t_{4}\right)\right] d t_{4}-\frac{1}{8} \int_{0}^{t}\left[\iint_{0}^{t_{4}}\left[\int_{0}^{t_{3}}\left[\int_{0}^{t_{2}} A\left(t_{1}\right) d t_{1}, A\left(t_{2}\right)\right] d t_{2}, A\left(t_{3}\right)\right] d t_{3}, A\left(t_{4}\right)\right] d t_{4} .
\end{aligned}
$$

Since the matrix $A(t)$ in equation (2.1) belongs to the Lie algebra for all $t \geq 0$, any term of the Magnus expansion (2.12) belongs to the same Lie algebra. In other words all terms of Magnus series belongs to the same Lie algebra and any truncation of the Magnus series will also belong to the same Lie algebra. This implies that the exponential map of any truncation will necessarily stay in the corresponding Lie group. Therefore an approximated solution obtained by truncating the Magnus expansion at any order preserves the same qualitative features of the exact solution (Castellano et al. 2014).

Iserles, Marthinsen \& Nørsett (1999) introduced the 4th-order Magnus method MG4 and Iserles, Nørsett \& Rasmussen (2001) introduced the 6th-order Magnus method MG6, based on the Gauss-Legendre points. The algorithms for MG4 
and MG6 are as follows;

Fourth-order Magnus expansion method:

$$
\begin{aligned}
& A_{1}=A\left(t_{n}+\left(\frac{1}{2}-\frac{\sqrt{3}}{6}\right) h\right), \quad A_{2}=A\left(t_{n}+\left(\frac{1}{2}+\frac{\sqrt{3}}{6}\right) h\right), \\
& \Omega=\frac{1}{2} h\left(A_{1}+A_{2}\right)-\frac{\sqrt{3}}{12} h^{2}\left[A_{1}, A_{2}\right], \\
& Y_{n+1}=e^{\Omega} Y_{n} .
\end{aligned}
$$

Sixth-order Magnus expansion method:

$$
\begin{aligned}
& A_{1}=h A\left(t_{n}+\left(\frac{1}{2}-\frac{\sqrt{15}}{10}\right) h\right), A_{2}=h A\left(t_{n}+\frac{1}{2} h\right), A_{3}=h A\left(t_{n}+\left(\frac{1}{2}+\frac{\sqrt{15}}{10}\right) h\right) \\
& B_{1}=A_{2}, \quad B_{2}=\frac{\sqrt{15}}{3}\left(A_{3}-A_{1}\right), \quad B_{3}=\frac{10}{3}\left(A_{3}-2 A_{2}+A_{1}\right), \\
& \Omega=B_{1}+\frac{1}{12} B_{3}-\frac{1}{12}\left[B_{1}, B_{2}\right]+\frac{1}{240}\left[B_{2}, B_{3}\right]+\frac{1}{360}\left[B_{1},\left[B_{1}, B_{3}\right]\right]-\frac{1}{240}\left[B_{1},\left[B_{1}, B_{2}\right]\right]+\frac{1}{720}\left[B_{1},\left[B_{1},\left[B_{1}, B_{2}\right]\right]\right], \\
& Y_{n+1}=e^{\Omega} Y_{n} .
\end{aligned}
$$

If the matrix is a constant matrix then $A=A_{1}=A_{2}=A_{3}$ and all terms in the Magnus series $\Omega(t)=\sum_{k=0}^{\infty} H_{k}(t)$ are zero except for $H_{0}$. This yields $\Omega(t)=H_{0}(t)=A t$ and $Y(t)=\operatorname{Exp}(\Omega(t))=\operatorname{Exp}(A t)$ which gives the exact solution. As a result one can say that Magnus Expansion Method gives the exact solution when the coefficient matrix $A(t)$ is constant.

\section{The vibration of a mass on a spring}

There are various forces acting upon the mass on a spring. The force acts in the downward direction is positive and the force acts in the upward direction is negative.

\subsection{The forces acting upon the mass on a spring (Ross 1984)}

3.1.1 $F_{1}$, the force of gravity

Magnitude of the force of gravity is $m g$, where $g$ is the acceleration due to the gravity and $m$ is the mass,

$$
F_{1}=m g
$$

\subsection{2 $F_{2}$, the restoring force}

Magnitude of the restoring force of the spring is $k(x+l)$, where $k$ is the spring constant and $x+l$ is the amount of total elongation. In Fig.1(c) the mass is below the end of the unstreched spring, the restoring force is given by

$$
F_{2}=-k(x+l) .
$$


3.1.3 $F_{3}$, the damping force

The resisting force of the medium is called the damping force. The exact magnitude of the damping force isn't known but for small velocities the magnitude of the damping force is given by

$$
\left|F_{3}\right|=a\left|\frac{d x}{d t}\right|,
$$

where $a>0$ is the damping constant. Damping force acts in the opposite direction of the motion of the mass.

3.1.4 $F_{4}$, the external force

Let $F(t)$ be any external force that act upon the mass at time $t$,

$$
F_{4}=F(t)
$$

Applying Newton's second law, $F=m a$, where $F=F_{1}+F_{2}+F_{3}+F_{4}$ we get,

$$
m \frac{d^{2} x}{d t^{2}}=F_{1}+F_{2}+F_{3}+F_{4}
$$

Substituting the equations (24), (25), (26) and (27) into the equation (28) we obtain,

$$
m \frac{d^{2} x}{d t^{2}}=m g-k x-m g-a \frac{d x}{d t}+F(t),
$$

From the equation (29) we obtain the differential equation for the motion of the mass on a spring as follows;

$$
m \frac{d^{2} x}{d t^{2}}+a \frac{d x}{d t}+k x=F(t) .
$$

\subsection{Motion of a mass on a spring}

The motion is called undamped when $a=0$, otherwise it is called damped. If $F(t)=0$ for all $t$ the motion is called free, otherwise it is called forced (Ross 1984). Fig. 1 illustrates the motion of a mass on a spring. 


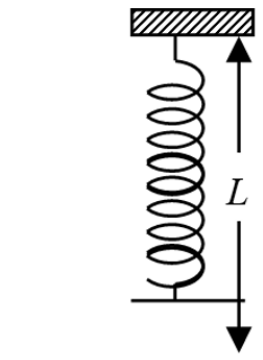

(a) naturel length $L$

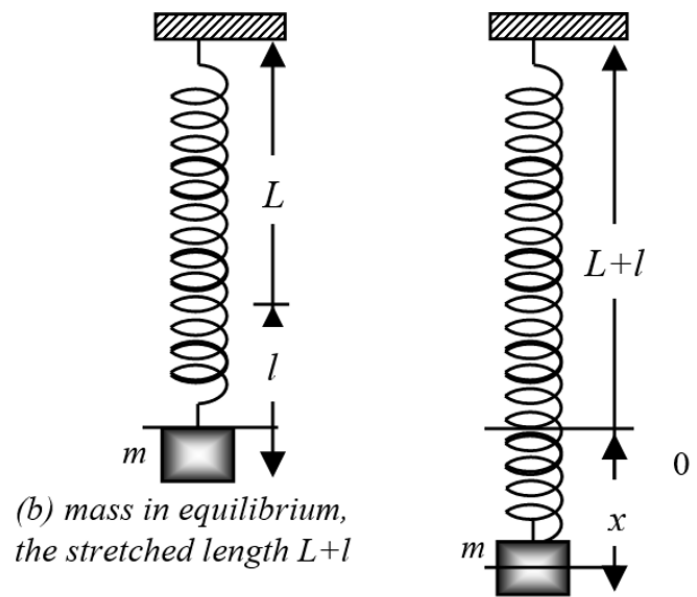

(c) mass distance $x$ below equilibrium position, spring stretched to length $L+l+x$

Fig. 1: The motion of a mass on a spring (Ross 1984).

\subsection{Free undamped motion}

In free undamped motion both the damping constant $a=0$ and the external force $F(t)=0$ in the equation (30). The differential equation (30) reduces to

$$
m \frac{d^{2} x}{d t^{2}}+k x=0,
$$

where $m$ is the mass and $k$ is the spring constant. Dividing the equation by $m$ and replacing $k / m$ by $\lambda^{2}$, equation (31) takes the form,

$$
\frac{d^{2} x}{d t^{2}}+\lambda^{2} x=0
$$

\subsection{Free damped motion}

In free damped motion damping constant $a \neq 0$ and the external force $F(t)=0$ in the equation (31). The differential equation (31) reduces to

$$
m \frac{d^{2} x}{d t^{2}}+a \frac{d x}{d t}+k x=0 .
$$

where $m$ is the mass and $k$ is the spring constant. Dividing the equation by $m$ and replacing $k / m$ by $\lambda^{2}$ and $a / m$ and $2 b$ respectively, equation (3.10) takes the form,

$$
\frac{d^{2} x}{d t^{2}}+2 b \frac{d x}{d t}+\lambda^{2} x=0 .
$$

Since $a$ and $m$ are positive, $b$ is positive. There are three cases in free damped motion (Ross 1984).

Case 1. Damped, Oscillatory Motion: In this case $b<\lambda$. The exact solution of the equation (34) has the oscillatory character and represents an oscillatory motion.

Case 2. Critical Damping: In this case $b=\lambda$. The motion is no longer oscillatory.

Case 3. Overcritical Damping: In this case $b>\lambda$. The exact solution of the equation (34) is not oscillatory. 


\subsubsection{Forced motion}

In forced motion the external force $F(t) \neq 0$ in the equation (3.7). We consider a periodic external impressed force $F$ defined by $F(t)=F_{1} \cos \omega t$ where $F$ and $\omega$ are constants. Then the differential equation (30) reduces to

$$
m \frac{d^{2} x}{d t^{2}}+a \frac{d x}{d t}+k x=F_{1} \cos \omega t
$$

where $m$ is the mass and $k$ is the spring constant (Ross 1984).

\section{Numerical examples}

In the higher order differential equations the diemonsion of the coefficient matrix increases i.e., in a third order homogeneous differential equation $\operatorname{dim}(A(t))=3$. We only consider second order ODEs, since in the vibration of a mass on a spring only second order differential equations occur.

In this section, six examples are investigated for various cases to illustrate the efficiency and the accuracy of MG4 and MG6. Since the coefficient matrices $A(t)$ are constant Magnus series Method gives the exact solution in examples 1-4.

Example 1. Consider free undamped motion (Ross 1984),

$$
\frac{d^{2} x}{d t^{2}}+64 x=0
$$

with the initial conditions,

$$
x(0)=1 / 4, x^{\prime}(0)=0 .
$$

By using the following transformation (Blanes et al. 2014),

$$
x=x_{1}, \quad x_{1}^{\prime}=x_{2},
$$

the equation (36) yields the Lie-type matrix equation,

$$
\left[\begin{array}{l}
x_{1} \\
x_{2}
\end{array}\right]^{\prime}=\left[\begin{array}{cc}
0 & 1 \\
-64 & 0
\end{array}\right]\left[\begin{array}{l}
x_{1} \\
x_{2}
\end{array}\right]
$$

where prime denotes derivative.

Let's call $\left[\begin{array}{l}x_{1} \\ x_{2}\end{array}\right]$ as $X$ and $\left[\begin{array}{cc}0 & 1 \\ -64 & 0\end{array}\right]$ matrix as $A$, then equation (4.1) takes the form,

$$
X^{\prime}=A X
$$

which is the linear Lie-type equation. Since the coefficient matrices $A(t)$ is constant Magnus series Method gives the exact solution.

Example 2. Consider free damped motion (Ross 1984),

$$
\frac{d^{2} x}{d t^{2}}+4 \frac{d x}{d t}+16 x=0
$$

with the initial conditions,

$$
x(0)=1 / 2, \quad x^{\prime}(0)=0 .
$$


By using the following transformation,

$$
x=x_{1}, \quad x_{1}^{\prime}=x_{2},
$$

the equation (41) yields the Lie-type matrix equation,

$$
\left[\begin{array}{l}
x_{1} \\
x_{2}
\end{array}\right]^{\prime}=\left[\begin{array}{cc}
0 & 1 \\
-64 & -4
\end{array}\right]\left[\begin{array}{l}
x_{1} \\
x_{2}
\end{array}\right]
$$

Let's call $\left[\begin{array}{l}x_{1} \\ x_{2}\end{array}\right]$ as $X$ and $\left[\begin{array}{cc}0 & 1 \\ -64 & -4\end{array}\right]$ matrix as $A$, then equation (41) takes the form,

$$
X^{\prime}=A X
$$

Since the coefficient matrices $A(t)$ is constant Magnus series Method gives the exact solution.

Example 3. Consider free critical damping (Ross 1984),

$$
\frac{d^{2} x}{d t^{2}}+8 \frac{d x}{d t}+16 x=0
$$

with the initial conditions,

$$
x(0)=1 / 2, \quad x^{\prime}(0)=0 .
$$

By using the following transformation,

$$
x=x_{1}, \quad x_{1}^{\prime}=x_{2},
$$

the equation (46) yields the Lie-type matrix equation,

$$
\left[\begin{array}{l}
x_{1} \\
x_{2}
\end{array}\right]^{\prime}=\left[\begin{array}{cc}
0 & 1 \\
-16 & -8
\end{array}\right]\left[\begin{array}{l}
x_{1} \\
x_{2}
\end{array}\right]
$$

where prime denotes derivative.

Let's call $\left[\begin{array}{l}x_{1} \\ x_{2}\end{array}\right]$ as $X$ and $\left[\begin{array}{cc}0 & 1 \\ -16 & -8\end{array}\right]$ matrix as $A$, then equation (46) takes the form,

$$
X^{\prime}=A X
$$

Since the coefficient matrices $A(t)$ is constant Magnus series Method gives the exact solution.

Example 4. Consider free overcritical damping (Ross 1984),

$$
\frac{d^{2} x}{d t^{2}}+10 \frac{d x}{d t}+16 x=0
$$

with the initial conditions,

$$
x(0)=1 / 2, \quad x^{\prime}(0)=0 .
$$

By using the following transformation,

$$
x=x_{1}, \quad x_{1}^{\prime}=x_{2},
$$


the equation (51) yields the Lie-type matrix equation,

$$
\left[\begin{array}{l}
x_{1} \\
x_{2}
\end{array}\right]^{\prime}=\left[\begin{array}{cc}
0 & 1 \\
-16 & -10
\end{array}\right]\left[\begin{array}{l}
x_{1} \\
x_{2}
\end{array}\right]
$$

Let's call $\left[\begin{array}{l}x_{1} \\ x_{2}\end{array}\right]$ as $X$ and $\left[\begin{array}{cc}0 & 1 \\ -16 & -10\end{array}\right]$ matrix as $A$, then equation (51) takes the form,

$$
X^{\prime}=A X
$$

Since the coefficient matrices $A(t)$ is constant Magnus series Method gives the exact solution.

Example 5. Consider forced motion with damping (Boyce \& DiPrima, 2001),

$$
\frac{d^{2} x}{d t^{2}}+\frac{1}{8} \frac{d x}{d t}+x=3 \cos 2 t
$$

with the initial conditions,

$$
x(0)=2, x^{\prime}(0)=0
$$

By using the following transformation (Blanes et al. 2014),

$$
x=x_{1}, \quad x_{1}^{\prime}=x_{2},
$$

the equation (51) yields the Lie-type matrix equation,

$$
\left[\begin{array}{c}
x_{1} \\
x_{2} \\
1
\end{array}\right]^{\prime}=\left[\begin{array}{ccc}
0 & 1 & 0 \\
-1 & -1 / 8 & 3 \cos 2 t \\
0 & 0 & 0
\end{array}\right]\left[\begin{array}{c}
x_{1} \\
x_{2} \\
1
\end{array}\right]
$$

Let's call $\left[\begin{array}{c}x_{1} \\ x_{2} \\ 1\end{array}\right]$ as $X$ and $\left[\begin{array}{ccc}0 & 1 & 0 \\ -1 & -1 / 8 & 3 \cos 2 t \\ 0 & 0 & 0\end{array}\right]$ matrix as $A$, then equation (56) takes the form,

$$
X^{\prime}=A X
$$

Then equation (60) is solved by MG4, MG6, RK4 and RK6. Tables 1-8. compare exact solution and obtained results and Tables 9,10. compare the absolute errors of the obtained aproximations of equation $(56)$ for the time interval $(0,10)$ with the step size $h=1 / 100$ respectively. Fig. 2-9 illustrate MG4, MG6, RK4 and RK6 solutions and the absolute errors for the time interval with the step size $h=1 / 100(0,10)$. 


\begin{tabular}{llll}
\hline $\mathrm{t}$ & Exact Solution & MG4 Solution & Absolute Error \\
\hline 0 & 2 & 2 & 0 \\
1 & 2.029400775781917 & 2.029400775747975 & $3.39426 \times 10^{-11}$ \\
2 & -0.485949674954965 & -0.485949674929983 & $2.49818 \times 10^{-11}$ \\
3 & -3.428483794322376 & -3.428483794253949 & $6.84266 \times 10^{-11}$ \\
4 & -1.323679341941148 & -1.323679341963859 & $2.27105 \times 10^{-11}$ \\
5 & 1.373677792211145 & 1.373677792173146 & $3.79987 \times 10^{-11}$ \\
6 & 1.081560844958590 & 1.081560844972373 & $1.37836 \times 10^{-11}$ \\
7 & 1.429350995372519 & 1.429350995347525 & $2.49942 \times 10^{-11}$ \\
8 & 0.704195131142868 & 0.704195131133206 & $9.66283 \times 10^{-12}$ \\
9 & -2.253898675340495 & -2.253898675278972 & $6.15232 \times 10^{-11}$ \\
10 & -1.696862886577910 & -1.696862886575667 & $2.24332 \times 10^{-12}$
\end{tabular}

Table 1: Comparison of exact solution and MG4 approximation for $x(t)$ for the time interval $(0,10)$ with the step size $h=1 / 100$ of equation (56).

\begin{tabular}{llll}
\hline $\mathrm{t}$ & Exact Solution & RK4 Solution & Absolute Error \\
\hline 0 & 2 & 0 & 0 \\
1 & 2.029400775781917 & 2.029400773409707 & $2.37221 \times 10^{-9}$ \\
2 & -0.485949674954965 & -0.485949668860424 & $6.09454 \times 10^{-9}$ \\
3 & -3.428483794322376 & -3.428483781785366 & $1.25370 \times 10^{-8}$ \\
4 & -1.323679341941148 & -1.323679351276856 & $9.33570 \times 10^{-9}$ \\
5 & 1.373677792211145 & 1.373677774141127 & $1.80700 \times 10^{-8}$ \\
6 & 1.081560844958590 & 1.081560843692483 & $1.26610 \times 10^{-9}$ \\
7 & 1.429350995372519 & 1.429351003899459 & $8.52693 \times 10^{-9}$ \\
8 & 0.704195131142868 & 0.704195143765409 & $1.26225 \times 10^{-8}$ \\
9 & -2.253898675340495 & -2.253898660698547 & $1.46419 \times 10^{-8}$ \\
10 & -1.696862886577910 & -1.696862895416944 & $8.83903 \times 10^{-9}$
\end{tabular}

Table 2: Comparison of exact solution and RK4 approximation for $x(t)$ for the time interval $(0,10)$ with the step size $h=1 / 100$ of equation (56).

\begin{tabular}{llll}
\hline $\mathrm{t}$ & Exact Solution & MG4 Solution & Absolute Error \\
\hline 0 & 0 & 0 & 0 \\
1 & -0.70657932509473 & -0.70657932518618 & $9.14421 \times 10^{-11}$ \\
2 & -3.95357238063817 & -3.95357238048988 & $1.48289 \times 10^{-10}$ \\
3 & -0.62434709733751 & -0.62434709733370 & $3.81505 \times 10^{-12}$ \\
4 & 3.77553137571673 & 3.77553137553797 & $1.78761 \times 10^{-10}$ \\
5 & 0.85002509487946 & 0.85002509496501 & $8.55518 \times 10^{-11}$ \\
6 & -0.43789592285876 & -0.43789592278766 & $7.11003 \times 10^{-11}$ \\
7 & 0.66097784752160 & 0.66097784739220 & $1.29393 \times 10^{-10}$ \\
8 & -2.51458679792127 & -2.51458679783686 & $8.44124 \times 10^{-11}$ \\
9 & -2.02605871092331 & -2.02605871082897 & $9.43414 \times 10^{-11}$ \\
10 & 2.79996360672381 & 2.79996360655452 & $1.69289 \times 10^{-10}$
\end{tabular}

Table 3: Comparison of exact solution and MG4 approximation for $x^{\prime}(t)$ for the time interval $(0,10)$ with the step size $h=1 / 100$ of equation (45). 


\begin{tabular}{llll}
\hline $\mathrm{t}$ & Exact Solution & RK4 Solution & Absolute Error \\
\hline 0 & 0 & $-1.40607 \times 10^{-19}$ & $1.40607 \times 10^{-19}$ \\
1 & -0.70657932509473 & -0.70657933027619 & $5.18145 \times 10^{-9}$ \\
2 & -3.95357238063817 & -3.95357237069818 & $9.93998 \times 10^{-9}$ \\
3 & -0.62434709733751 & -0.62434710542397 & $8.08645 \times 10^{-9}$ \\
4 & 3.77553137571673 & 3.77553136162707 & $1.40897 \times 10^{-8}$ \\
5 & 0.85002509487946 & 0.85002511504699 & $2.01675 \times 10^{-8}$ \\
6 & -0.43789592285876 & -0.43789590592604 & $1.69327 \times 10^{-8}$ \\
7 & 0.66097784752160 & 0.66097784999469 & $2.47309 \times 10^{-9}$ \\
8 & -2.51458679792127 & -2.51458679695384 & $9.67436 \times 10^{-10}$ \\
9 & -2.02605871092331 & -2.02605872071632 & $9.79301 \times 10^{-9}$ \\
10 & 2.79996360672381 & 2.79996357327951 & $3.34443 \times 10^{-8}$
\end{tabular}

Table 4: Comparison of exact solution and RK4 approximation for $x^{\prime}(t)$ for the time interval $(0,10)$ with the step size $h=1 / 100$ of equation (56).

\begin{tabular}{llll}
\hline $\mathrm{t}$ & Exact Solution & MG6 Solution & Absolute Error \\
\hline 0 & 2 & 2 & 0 \\
1 & 2.029400775781917 & 2.029400775781906 & $1.15463 \times 10^{-14}$ \\
2 & -0.485949674954965 & -0.485949674954977 & $1.18793 \times 10^{-14}$ \\
3 & -3.428483794322376 & -3.428483794322334 & $4.17443 \times 10^{-14}$ \\
4 & -1.323679341941148 & -1.323679341941079 & $6.94999 \times 10^{-14}$ \\
5 & 1.373677792211145 & 1.373677792211128 & $1.68753 \times 10^{-14}$ \\
6 & 1.081560844958590 & 1.081560844958511 & $7.88258 \times 10^{-14}$ \\
7 & 1.429350995372519 & 1.429350995372449 & $7.06101 \times 10^{-14}$ \\
8 & 0.704195131142868 & 0.704195131142861 & $7.10542 \times 10^{-14}$ \\
9 & -2.253898675340495 & -2.253898675340423 & $7.14983 \times 10^{-14}$ \\
10 & -1.696862886577910 & -1.696862886577779 & $1.30784 \times 10^{-13}$
\end{tabular}

Table 5: Comparison of exact solution and MG6 approximation for $x(t)$ for the time interval $(0,10)$ with the step size $h=1 / 100$ of equation (56).

\begin{tabular}{llll}
\hline $\mathrm{t}$ & Exact Solution & RK6 Solution & Absolute Error \\
\hline 0 & 2 & 2 & 0 \\
1 & 2.029400775781917 & 2.029400775782261 & $3.43280 \times 10^{-13}$ \\
2 & -0.485949674954965 & -0.485949674963123 & $8.15880 \times 10^{-12}$ \\
3 & -3.428483794322376 & -3.428483794329231 & $6.85496 \times 10^{-12}$ \\
4 & -1.323679341941148 & -1.323679341979057 & $3.79083 \times 10^{-11}$ \\
5 & 1.373677792211145 & 1.373677792205894 & $5.25091 \times 10^{-12}$ \\
6 & 1.081560844958590 & 1.081560844955451 & $3.13860 \times 10^{-12}$ \\
7 & 1.429350995372519 & 1.429350995355398 & $1.71214 \times 10^{-11}$ \\
8 & 0.704195131142868 & 0.704195131092251 & $5.06177 \times 10^{-11}$ \\
9 & -2.253898675340495 & -2.253898675338253 & $2.24131 \times 10^{-12}$ \\
10 & -1.696862886577910 & -1.696862886501658 & $7.62514 \times 10^{-11}$
\end{tabular}

Table 6: Comparison of exact solution and RK6 approximation for $x(t)$ for the time interval $(0,10)$ with the step size $h=1 / 100$ of equation (56). 
101 EISA M. Basbuk, A. Eryilmaz and M. T. Atay: Vibration analysis of a mass on a spring by means of magnus...

\begin{tabular}{llll}
\hline $\mathrm{t}$ & Exact Solution & MG6 Solution & Absolute Error \\
\hline 0 & 0 & 0 & 0 \\
1 & -0.70657932509473 & -0.70657932509472 & $1.27676 \times 10^{-14}$ \\
2 & -3.95357238063817 & -3.95357238063815 & $2.08722 \times 10^{-14}$ \\
3 & -0.62434709733751 & -0.62434709733748 & $2.59792 \times 10^{-14}$ \\
4 & 3.77553137571673 & 3.77553137571668 & $5.28466 \times 10^{-14}$ \\
5 & 0.85002509487946 & 0.85002509487938 & $8.08242 \times 10^{-14}$ \\
6 & -0.43789592285876 & -0.43789592285877 & $1.44884 \times 10^{-14}$ \\
7 & 0.66097784752160 & 0.66097784752166 & $6.25056 \times 10^{-14}$ \\
8 & -2.51458679792127 & -2.51458679792119 & $8.30447 \times 10^{-14}$ \\
9 & -2.02605871092331 & -2.02605871092325 & $6.61693 \times 10^{-14}$ \\
10 & 2.79996360672381 & 2.79996360672376 & $5.06262 \times 10^{-14}$
\end{tabular}

Table 7: Comparison of exact solution and MG6 approximation for $x^{\prime}(t)$ for the time interval $(0,10)$ with the step size $h=1 / 100$ of equation (56).

\begin{tabular}{llll}
\hline $\mathrm{t}$ & Exact Solution & RK6 Solution & Absolute Error \\
\hline 0 & 0 & $-1.04185 \times 10^{-19}$ & $1.04185 \times 10^{-19}$ \\
1 & -0.70657932509473 & -0.70657932469200 & $4.02728 \times 10^{-10}$ \\
2 & -3.95357238063817 & -3.95357237958909 & $1.04908 \times 10^{-9}$ \\
3 & -0.62434709733751 & -0.62434709767115 & $3.33644 \times 10^{-10}$ \\
4 & 3.77553137571673 & 3.77553137915158 & $3.43485 \times 10^{-9}$ \\
5 & 0.85002509487946 & 0.85002509838032 & $3.50086 \times 10^{-9}$ \\
6 & -0.43789592285876 & -0.43789592155104 & $1.30772 \times 10^{-9}$ \\
7 & 0.66097784752160 & 0.66097784760867 & $8.70735 \times 10^{-11}$ \\
8 & -2.51458679792127 & -2.51458679431495 & $3.60633 \times 10^{-9}$ \\
9 & -2.02605871092331 & -2.02605870759639 & $3.32692 \times 10^{-9}$ \\
10 & 2.79996360672381 & 2.79996360722260 & $4.98793 \times 10^{-10}$
\end{tabular}

Table 8: Comparison of exact solution and RK6 approximation for $x^{\prime}(t)$ for the time interval $(0,10)$ with the step size $h=1 / 100$ of equation (56).

\begin{tabular}{lllll}
\hline $\mathrm{t}$ & MG6 & RK6 & MG4 & RK4 \\
\hline 0 & 0 & 0 & 0 & 0 \\
1 & $1.15463 \times 10^{-14}$ & $3.43280 \times 10^{-13}$ & $3.39426 \times 10^{-11}$ & $2.37221 \times 10^{-9}$ \\
2 & $1.18793 \times 10^{-14}$ & $8.15880 \times 10^{-12}$ & $2.49818 \times 10^{-11}$ & $6.09454 \times 10^{-9}$ \\
3 & $4.17443 \times 10^{-14}$ & $6.85496 \times 10^{-12}$ & $6.84266 \times 10^{-11}$ & $1.25370 \times 10^{-8}$ \\
4 & $6.94999 \times 10^{-14}$ & $3.79083 \times 10^{-11}$ & $2.27105 \times 10^{-11}$ & $9.33570 \times 10^{-9}$ \\
5 & $1.68753 \times 10^{-14}$ & $5.25091 \times 10^{-12}$ & $3.79987 \times 10^{-11}$ & $1.80700 \times 10^{-8}$ \\
6 & $7.88258 \times 10^{-14}$ & $3.13860 \times 10^{-12}$ & $1.37836 \times 10^{-11}$ & $1.26610 \times 10^{-9}$ \\
7 & $7.06101 \times 10^{-14}$ & $1.71214 \times 10^{-11}$ & $2.49942 \times 10^{-11}$ & $8.52693 \times 10^{-9}$ \\
8 & $7.10542 \times 10^{-14}$ & $5.06177 \times 10^{-11}$ & $9.66283 \times 10^{-12}$ & $1.26225 \times 10^{-8}$ \\
9 & $7.14983 \times 10^{-14}$ & $2.24131 \times 10^{-12}$ & $6.15232 \times 10^{-11}$ & $1.46419 \times 10^{-8}$ \\
10 & $1.30784 \times 10^{-13}$ & $7.62514 \times 10^{-11}$ & $2.24332 \times 10^{-12}$ & $8.83903 \times 10^{-9}$
\end{tabular}

Table 9: Comparison of absolute errors of MG4, MG6, RK4 and RK6 solutions for $x(t)$ for the time interval $(0,10)$ with the step size $h=1 / 100$ of equation (56). 


\begin{tabular}{lllll}
\hline $\mathrm{t}$ & MG6 & RK6 & MG4 & RK4 \\
\hline 0 & 0 & $1.04185 \times 10^{-19}$ & 0 & $1.40607 \times 10^{-19}$ \\
1 & $1.27676 \times 10^{-14}$ & $4.02728 \times 10^{-10}$ & $9.14421 \times 10^{-11}$ & $5.18145 \times 10^{-9}$ \\
2 & $2.08722 \times 10^{-14}$ & $1.04908 \times 10^{-9}$ & $1.48289 \times 10^{-10}$ & $9.93998 \times 10^{-9}$ \\
3 & $2.59792 \times 10^{-14}$ & $3.33644 \times 10^{-10}$ & $3.81505 \times 10^{-12}$ & $8.08645 \times 10^{-9}$ \\
4 & $5.28466 \times 10^{-14}$ & $3.43485 \times 10^{-9}$ & $1.78761 \times 10^{-10}$ & $1.40897 \times 10^{-8}$ \\
5 & $8.08242 \times 10^{-14}$ & $3.50086 \times 10^{-9}$ & $8.55518 \times 10^{-11}$ & $2.01675 \times 10^{-8}$ \\
6 & $1.44884 \times 10^{-14}$ & $1.30772 \times 10^{-9}$ & $7.11003 \times 10^{-11}$ & $1.69327 \times 10^{-8}$ \\
7 & $6.25056 \times 10^{-14}$ & $8.70735 \times 10^{-11}$ & $1.29393 \times 10^{-10}$ & $2.47309 \times 10^{-9}$ \\
8 & $8.30447 \times 10^{-14}$ & $3.60633 \times 10^{-9}$ & $8.44124 \times 10^{-11}$ & $9.67436 \times 10^{-10}$ \\
9 & $6.61693 \times 10^{-14}$ & $3.32692 \times 10^{-9}$ & $9.43414 \times 10^{-11}$ & $9.79301 \times 10^{-9}$ \\
10 & $5.06262 \times 10^{-14}$ & $4.98793 \times 10^{-10}$ & $1.69289 \times 10^{-10}$ & $3.34443 \times 10^{-8}$
\end{tabular}

Table 10: Comparison of absolute errors of MG4, MG6, RK4 and RK6 solutions for $x^{\prime}(t)$ for the time interval $(0,10)$ with the step size $h=1 / 100$ of equation (56).

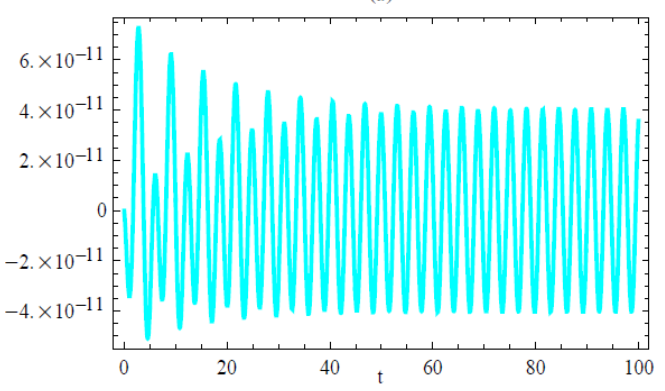

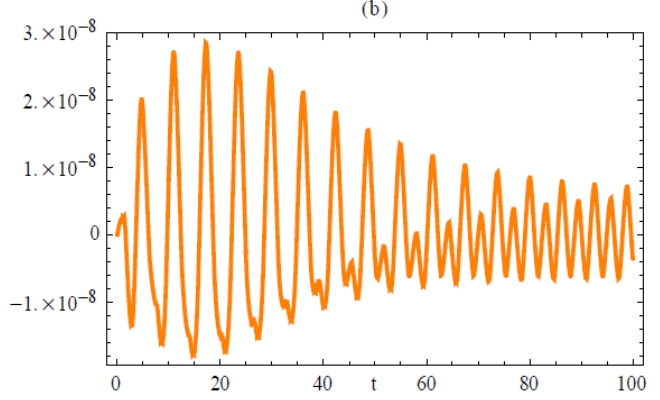

Fig. 2: Error graphics for $x(t)$ (a) MG4, (b) RK4 solution of equation (56).
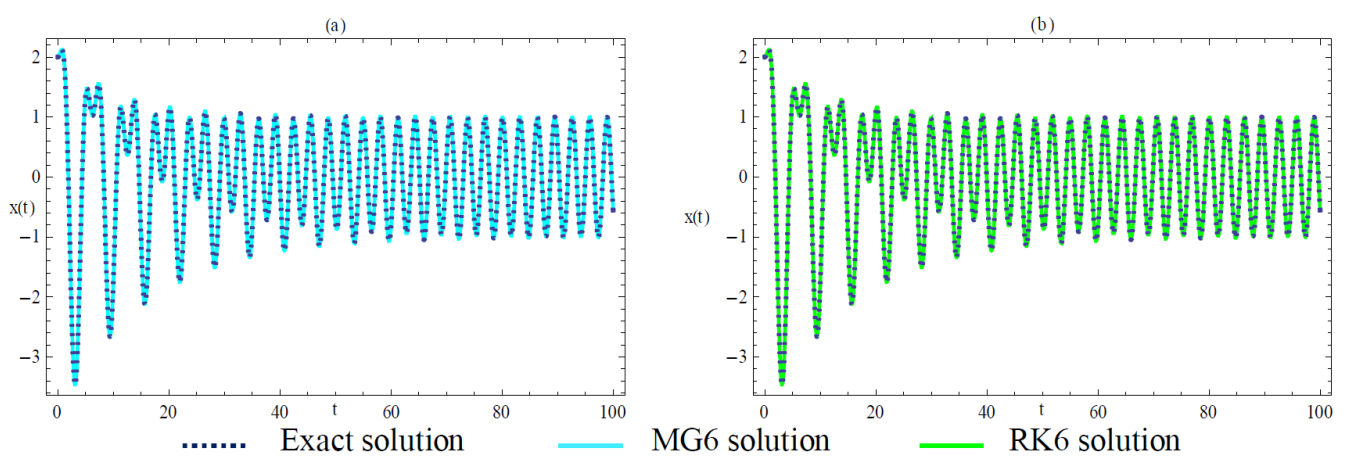

Fig. 3: Comparison of (a) MG4, (b) RK4 with exact solution for $x(t)$ in equation (56). 

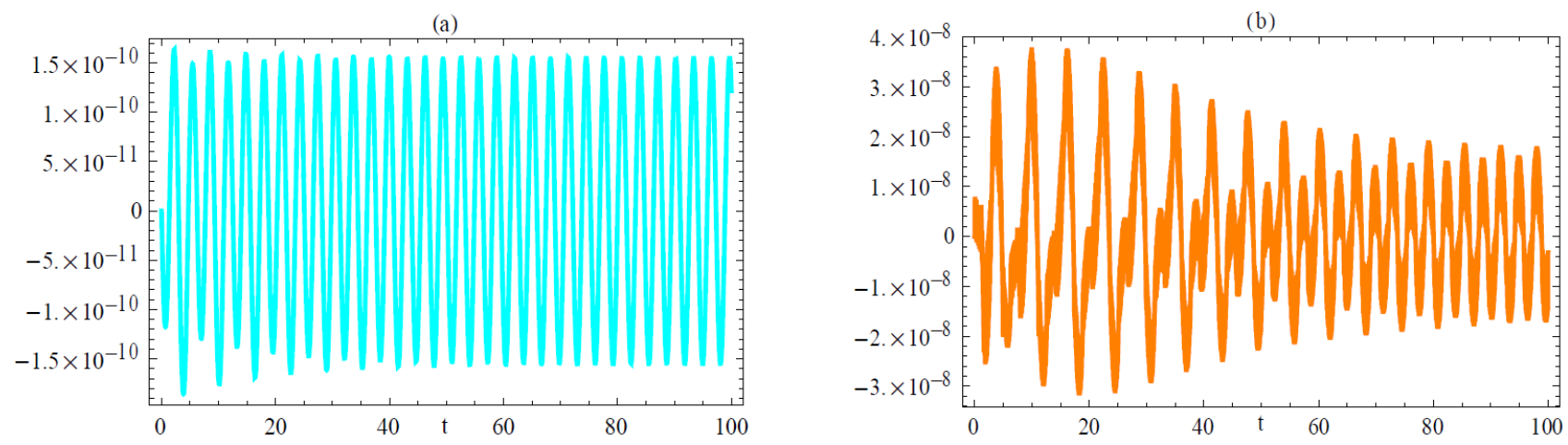

Fig. 4: Error graphics for $x^{\prime}(t)$ (a) MG4, (b) RK4 solution of equation (56).
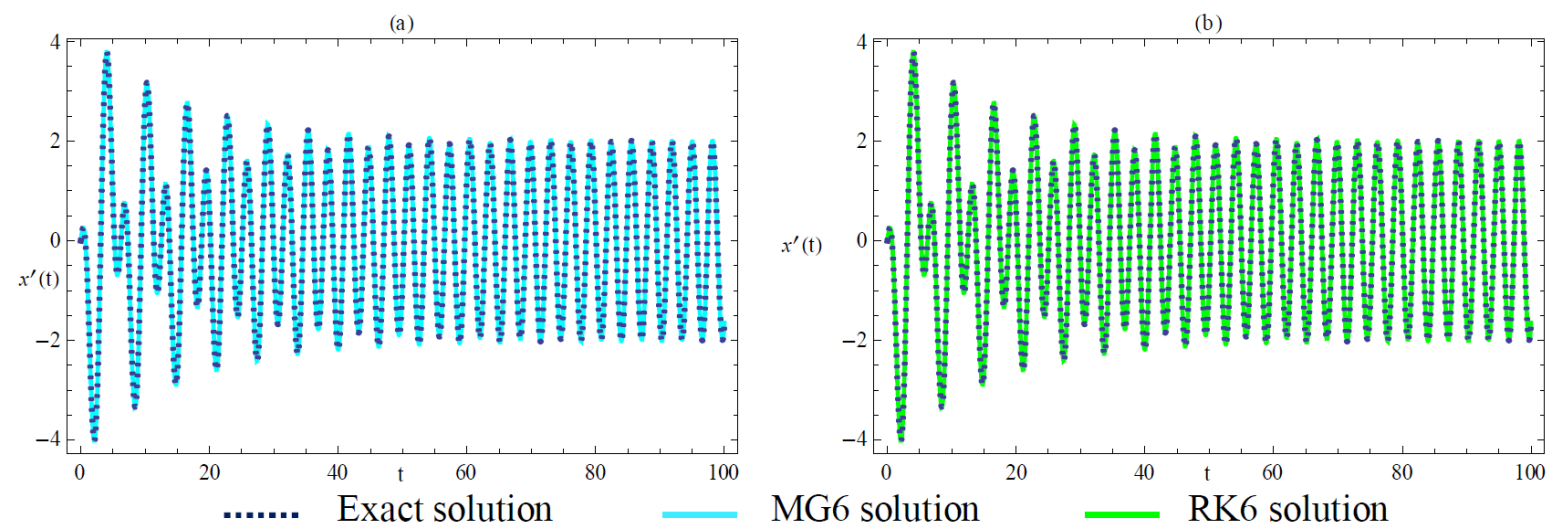

Fig. 5: Comparison of (a) MG4, (b) RK4 with exact solution for $x^{\prime}(t)$ in equation (56).
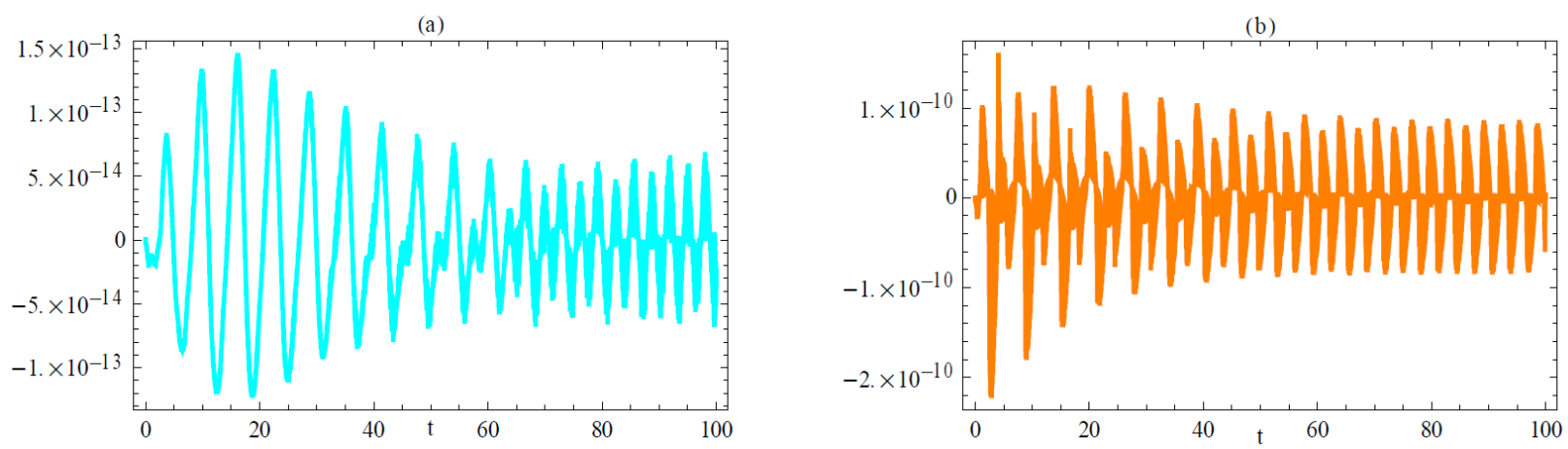

Fig. 6: Error graphics for $x(t)$ (a) MG6, (b) RK6 solution of equation (56). 
(a)

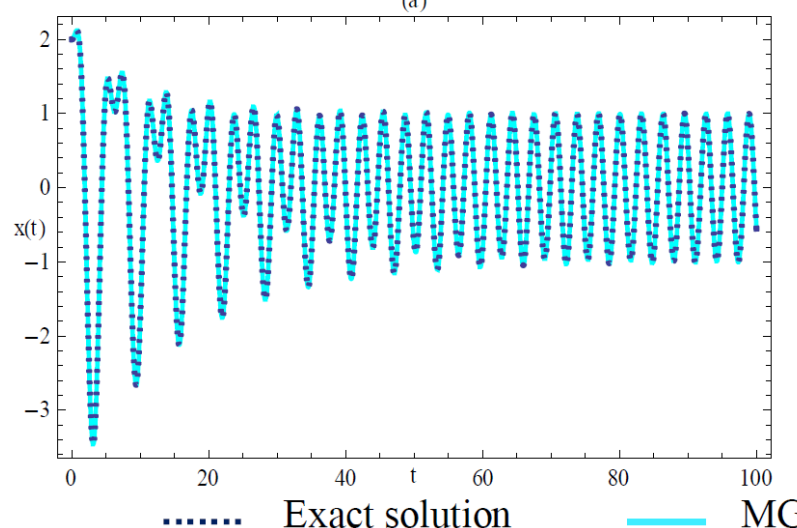

(b)

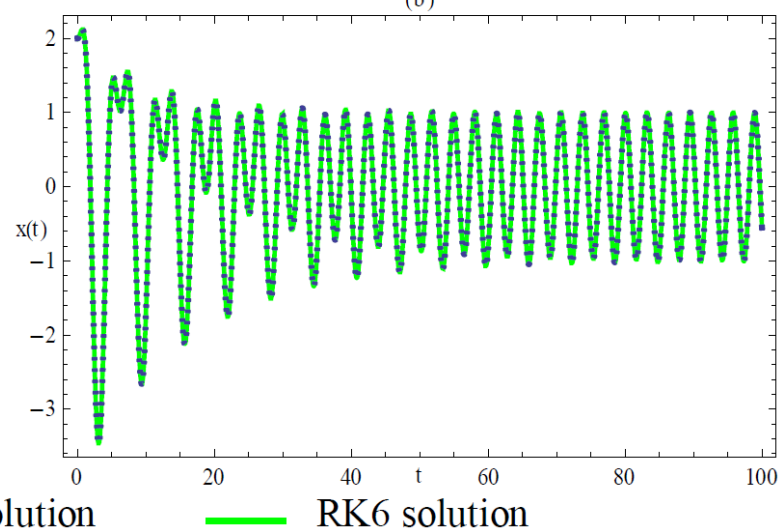

Fig. 7: Comparison of (a) MG6, (b) RK6 with exact solution for $x(t)$ in equation (56).

(a)

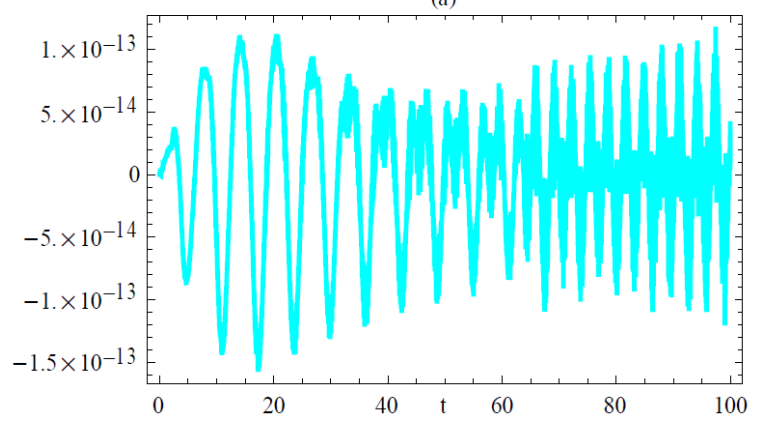

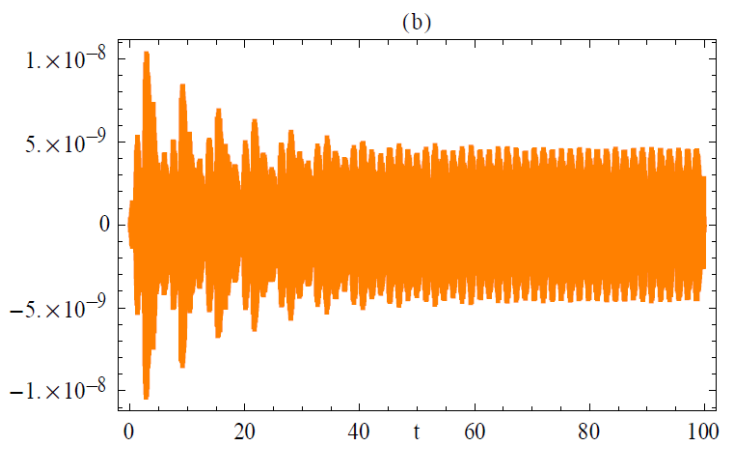

Fig. 8: Error graphics for $x^{\prime}(t)$ (a) MG6, (b) RK6 solution of equation (56).

(a)

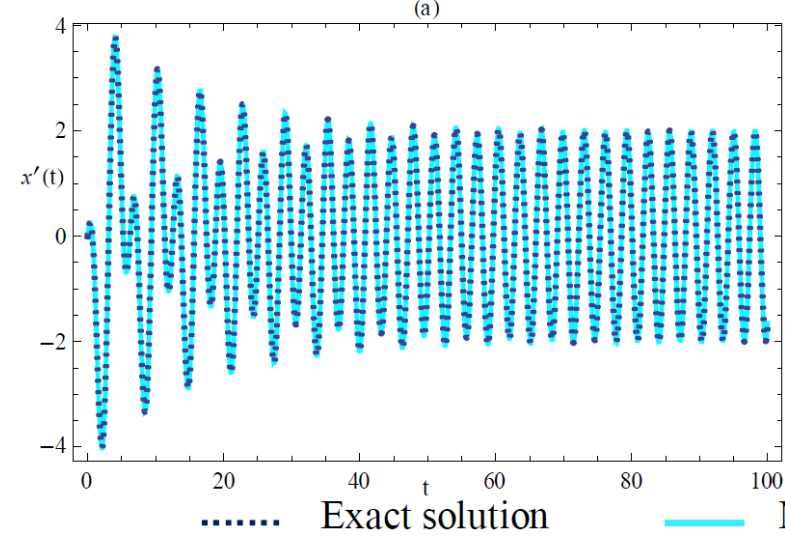

(b)

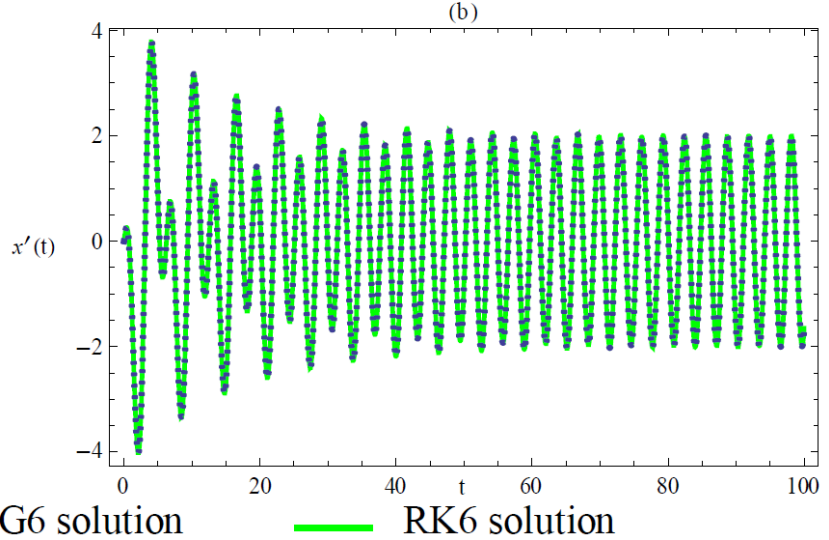

Fig. 9: Comparison of (a) MG6, (b) RK6 with exact solution for $x^{\prime}(t)$ in equation (56). 


\begin{tabular}{llll}
\hline $\mathrm{t}$ & Exact Solution & MG4 Solution & Absolute Error \\
\hline 0 & 0 & 0 & 0 \\
1 & 0.210367746201974 & 0.210367746195660 & $6.31398 \times 10^{-12}$ \\
2 & 0.454648713412840 & 0.454648713399192 & $1.36483 \times 10^{-11}$ \\
3 & 0.105840006044900 & 0.105840006041720 & $3.17973 \times 10^{-12}$ \\
4 & -0.756802495307928 & -0.756802495285208 & $2.27196 \times 10^{-11}$ \\
5 & -1.198655343328923 & -1.198655343292929 & $3.59939 \times 10^{-11}$ \\
6 & -0.419123247298388 & -0.419123247285797 & $1.25912 \times 10^{-11}$ \\
7 & 1.149726547757880 & 1.149726547723353 & $3.45277 \times 10^{-11}$ \\
8 & 1.978716493246763 & 1.978716493187326 & $5.94373 \times 10^{-11}$ \\
9 & 0.927266591793952 & 0.927266591766087 & $2.78653 \times 10^{-11}$ \\
10 & -1.360052777223424 & -1.360052777182569 & $4.08553 \times 10^{-11}$
\end{tabular}

Table 11: Comparison of exact solution and MG4 approximation for $x(t)$ for the time interval $(0,10)$ with the step size $h=1 / 100$ of equation (61).

Example 6. Consider forced motion without damping (Boyce \& DiPrima, 2001),

$$
\frac{d^{2} x}{d t^{2}}+x=0.5 \cos t
$$

with the initial conditions,

$$
x(0)=0, \quad x^{\prime}(0)=0 .
$$

By using the following transformation (Blanes et al. 2014),

$$
x=x_{1}, \quad x_{1}^{\prime}=x_{2}
$$

the equation (61) yields the Lie-type matrix equation,

$$
\left[\begin{array}{c}
x_{1} \\
x_{2} \\
1
\end{array}\right]^{\prime}=\left[\begin{array}{ccc}
0 & 1 & 0 \\
-1 & 0 & 0.5 \cos t \\
0 & 0 & 0
\end{array}\right]\left[\begin{array}{c}
x_{1} \\
x_{2} \\
1
\end{array}\right]
$$

Let's call $\left[\begin{array}{c}x_{1} \\ x_{2} \\ 1\end{array}\right]$ as $X$ and $\left[\begin{array}{ccc}0 & 1 & 0 \\ -1 & 0 & 0.5 \cos t \\ 0 & 0 & 0\end{array}\right]$ as $A$, then equation (61) takes the form,

$$
X^{\prime}=A X
$$

Then equation (61) is solved by MG4, MG6, RK4 and RK6. Tables 11-18. compare exact solution and obtained results and Tables 19,20. compare the absolute errors of the obtained aproximations of equation (61) for the time interval $(0,10)$ with the step size $h=1 / 100$ respectively. Fig. 10-17. illustrate MG4, MG6, RK4 and RK6 solutions and the absolute errors for the time interval with the step size $h=1 / 100(0,10)$.

\section{Conclusions}

In this work, a reliable, efficient and structure preserving (Castellano et al. 2014) numerical algorithm based on the Magnus series expansion is applied to solve the differential equations which occur in the vibration of a mass on a spring. Some numerical examples are given to illustrate the validity and accuracy of this procedure. We investigate both the fourth and the sixth order Magnus Series method. Since the coefficient matrices $A(t)$ in the examples $1-4$ are constant 


\begin{tabular}{llll}
\hline $\mathrm{t}$ & Exact Solution & RK4 Solution & Absolute Error \\
\hline 0 & 0 & $1.75259 \times 10^{-23}$ & $1.75259 \times 10^{-23}$ \\
1 & 0.210367746201974 & 0.210367744329572 & $1.87240 \times 10^{-9}$ \\
2 & 0.454648713412840 & 0.454648710692657 & $2.72018 \times 10^{-9}$ \\
3 & 0.105840006044900 & 0.105840008380526 & $2.33563 \times 10^{-9}$ \\
4 & -0.756802495307928 & -0.756802486095031 & $9.21290 \times 10^{-9}$ \\
5 & -1.198655343328923 & -1.198655337051734 & $6.27719 \times 10^{-9}$ \\
6 & -0.419123247298388 & -0.419123255196930 & $7.89854 \times 10^{-9}$ \\
7 & 1.149726547757880 & 1.149726528525150 & $1.92327 \times 10^{-8}$ \\
8 & 1.978716493246763 & 1.978716482628974 & $1.06178 \times 10^{-8}$ \\
9 & 0.927266591793952 & 0.927266606801667 & $1.50077 \times 10^{-8}$ \\
10 & -1.360052777223424 & -1.360052744723619 & $3.24998 \times 10^{-8}$
\end{tabular}

Table 12: Comparison of exact solution and RK4 approximation for $x(t)$ for the time interval $(0,10)$ with the step size $h=1 / 100$ of equation (61).

\begin{tabular}{llll}
\hline $\mathrm{t}$ & Exact Solution & MG4 Solution & Absolute Error \\
\hline 0 & 0 & 0 & 0 \\
1 & 0.345443322669009 & 0.345443322664448 & $4.56063 \times 10^{-12}$ \\
2 & 0.019250938432849 & 0.019250938438545 & $5.69621 \times 10^{-12}$ \\
3 & -0.707214370435367 & -0.707214370413163 & $2.22036 \times 10^{-11}$ \\
4 & -0.842844244690593 & -0.842844244670509 & $2.00843 \times 10^{-11}$ \\
5 & 0.114846663163248 & 0.114846663153187 & $1.00612 \times 10^{-11}$ \\
6 & 1.370401555425817 & 1.370401555382737 & $4.30804 \times 10^{-11}$ \\
7 & 1.483575594780480 & 1.483575594740449 & $4.00304 \times 10^{-11}$ \\
8 & -0.043660505961381 & -0.043660505953252 & $8.12926 \times 10^{-12}$ \\
9 & -1.947013467930084 & -1.947013467868753 & $6.13301 \times 10^{-11}$ \\
10 & -2.233684100413473 & -2.233684100350112 & $6.33613 \times 10^{-11}$
\end{tabular}

Table 13: Comparison of exact solution and MG4 approximation for $x^{\prime}(t)$ for the time interval $(0,10)$ with the step size $\mathrm{h}=1 / 100$ of equation $(61)$.

\begin{tabular}{llll}
\hline $\mathrm{t}$ & Exact Solution & RK4 Solution & Absolute Error \\
\hline 0 & 0 & $1.16996 \times 10^{-20}$ & $1.16996 \times 10^{-20}$ \\
1 & 0.345443322669009 & 0.345443318227632 & $4.44138 \times 10^{-9}$ \\
2 & 0.019250938432849 & 0.019250941559083 & $3.12623 \times 10^{-9}$ \\
3 & -0.707214370435367 & -0.707214362702374 & $7.73299 \times 10^{-9}$ \\
4 & -0.842844244690593 & -0.842844238931988 & $5.75861 \times 10^{-9}$ \\
5 & 0.114846663163248 & 0.114846653001235 & $1.01620 \times 10^{-8}$ \\
6 & 1.370401555425817 & 1.370401536573017 & $1.88528 \times 10^{-8}$ \\
7 & 1.483575594780480 & 1.483575593825043 & $9.55437 \times 10^{-10}$ \\
8 & -0.043660505961381 & -0.043660485039647 & $2.09217 \times 10^{-8}$ \\
9 & -1.947013467930084 & -1.947013441188060 & $2.67420 \times 10^{-8}$ \\
10 & -2.233684100413473 & -2.233684093035864 & $7.37761 \times 10^{-9}$
\end{tabular}

Table 14: Comparison of exact solution and RK4 approximation for $x^{\prime}(t)$ for the time interval $(0,10)$ with the step size $h=1 / 100$ of equation (61).

Magnus Series Method gives the exact solution for all orders of Magnus Series Method. On the other hand for the nonhomogeneous equations MG6 gives beter results than MG4. So in the examples 5-6 we applied both MG4 and MG6 and compared the results with the exact solutions. Depending on a accuracy expectations and problem itself, MG4 or MG6 can be used alternatively. In general especially, in real problems such as vibration, oscillation or other mechanical phenomenon, MG4 provides good convergence in comparisons with exact solution As it can be seen from the results of this research, MG6 gives better numerical results for this problem but needs more computations. Also, smaller step size selections may provide better results with higher computational cost. 
107 EISA M. Basbuk, A. Eryilmaz and M. T. Atay: Vibration analysis of a mass on a spring by means of magnus...

\begin{tabular}{llll}
\hline $\mathrm{t}$ & Exact Solution & MG6 Solution & Absolute Error \\
\hline 0 & 0 & 0 & 0 \\
1 & 0.210367746201974 & 0.210367746201973 & $1.02696 \times 10^{-15}$ \\
2 & 0.454648713412840 & 0.454648713412835 & $4.88498 \times 10^{-15}$ \\
3 & 0.105840006044900 & 0.105840006044896 & $3.95517 \times 10^{-15}$ \\
4 & -0.756802495307928 & -0.756802495307919 & $9.21485 \times 10^{-15}$ \\
5 & -1.198655343328923 & -1.198655343328899 & $2.39808 \times 10^{-14}$ \\
6 & -0.419123247298388 & -0.419123247298375 & $1.31006 \times 10^{-14}$ \\
7 & 1.149726547757880 & 1.149726547757854 & $2.66454 \times 10^{-14}$ \\
8 & 1.978716493246763 & 1.978716493246705 & $5.81757 \times 10^{-14}$ \\
9 & 0.927266591793952 & 0.927266591793916 & $3.63043 \times 10^{-14}$ \\
10 & -1.360052777223424 & -1.360052777223381 & $4.32987 \times 10^{-14}$
\end{tabular}

Table 15: Comparison of exact solution and MG6 approximation for $x(t)$ for the time interval $(0,10)$ with the step size $h=1 / 100$ of equation (61).

\begin{tabular}{llll}
\hline $\mathrm{t}$ & Exact Solution & RK6 Solution & Absolute Error \\
\hline 0 & 0 & $1.62334 \times 10^{-23}$ & $1.62334 \times 10^{-23}$ \\
1 & 0.210367746201974 & 0.210367746203788 & $1.81477 \times 10^{-12}$ \\
2 & 0.454648713412840 & 0.454648713414681 & $1.84058 \times 10^{-12}$ \\
3 & 0.105840006044900 & 0.105840006037642 & $7.25758 \times 10^{-12}$ \\
4 & -0.756802495307928 & -0.756802495338657 & $3.07290 \times 10^{-11}$ \\
5 & -1.198655343328923 & -1.198655343336124 & $7.20091 \times 10^{-12}$ \\
6 & -0.419123247298388 & -0.419123247245486 & $5.29026 \times 10^{-11}$ \\
7 & 1.149726547757880 & 1.149726547816170 & $5.82896 \times 10^{-11}$ \\
8 & 1.978716493246763 & 1.978716493262211 & $1.54483 \times 10^{-11}$ \\
9 & 0.927266591793952 & 0.927266591742926 & $5.10261 \times 10^{-11}$ \\
10 & -1.360052777223424 & -1.360052777313749 & $9.03253 \times 10^{-11}$
\end{tabular}

Table 16: Comparison of exact solution and RK6 approximation for $x(t)$ for the time interval $(0,10)$ with the step size $h=1 / 100$ of equation (61).

\begin{tabular}{llll}
\hline $\mathrm{t}$ & Exact Solution & MG6 Solution & Absolute Error \\
\hline 0 & 0 & 0 & 0 \\
1 & 0.34544332266900 & 0.34544332266900 & $2.66453 \times 10^{-15}$ \\
2 & 0.01925093843284 & 0.01925093843284 & $2.99066 \times 10^{-15}$ \\
3 & -0.70721437043536 & -0.70721437043536 & $7.21644 \times 10^{-15}$ \\
4 & -0.84284424469059 & -0.84284424469057 & $1.64313 \times 10^{-14}$ \\
5 & 0.11484666316324 & 0.11484666316325 & $3.92741 \times 10^{-15}$ \\
6 & 1.37040155542581 & 1.37040155542579 & $2.68673 \times 10^{-14}$ \\
7 & 1.48357559478048 & 1.48357559478043 & $4.30766 \times 10^{-14}$ \\
8 & -0.04366050596138 & -0.04366050596138 & $8.32667 \times 10^{-15}$ \\
9 & -1.94701346793008 & -1.94701346793002 & $5.79536 \times 10^{-14}$ \\
10 & -2.23368410041347 & -2.23368410041338 & $8.52651 \times 10^{-14}$
\end{tabular}

Table 17: Comparison of exact solution and MG6 approximation for $x^{\prime}(t)$ for the time interval $(0,10)$ with the step size $h=1 / 100$ of equation (61).

As a result Magnus Series Expansion Method is an efficient, powerfull and accurate tool for vibration of a mass on a spring.

\section{References}

[1] Baye, D. \& Heenen P.H. 1973. A theoretical study of fast proton-atomic hydrogen scattering. J Phys B: At Mol. Phys. 6: 105-13. 


\begin{tabular}{llll}
\hline $\mathrm{t}$ & Exact Solution & RK6 Solution & Absolute Error \\
\hline 0 & 0 & $4.76456 \times 10^{-21}$ & $4.76456 \times 10^{-21}$ \\
1 & 0.34544332266900 & 0.34544332266260 & $6.40809 \times 10^{-12}$ \\
2 & 0.01925093843284 & 0.01925093781073 & $6.22117 \times 10^{-10}$ \\
3 & -0.70721437043536 & -0.70721437060022 & $1.64857 \times 10^{-10}$ \\
4 & -0.84284424469059 & -0.84284424461064 & $7.99509 \times 10^{-11}$ \\
5 & 0.11484666316324 & 0.11484666422816 & $1.06491 \times 10^{-9}$ \\
6 & 1.37040155542581 & 1.37040155635556 & $9.29750 \times 10^{-10}$ \\
7 & 1.48357559478048 & 1.48357559477285 & $7.62079 \times 10^{-12}$ \\
8 & -0.04366050596138 & -0.04366050503140 & $9.29978 \times 10^{-10}$ \\
9 & -1.94701346793008 & -1.94701346933480 & $1.40471 \times 10^{-10}$ \\
10 & -2.23368410041347 & -2.23368410042076 & $7.29638 \times 10^{-10}$
\end{tabular}

Table 18: Comparison of exact solution and RK6 approximation for $x^{\prime}(t)$ for the time interval $(0,10)$ with the step size $h=1 / 100$ of equation (61).

\begin{tabular}{lllll}
\hline $\mathrm{t}$ & MG6 & RK6 & MG4 & RK4 \\
\hline 0 & 0 & $1.62334 \times 10^{-23}$ & 0 & $1.75259 \times 10^{-23}$ \\
1 & $1.02696 \times 10^{-15}$ & $1.81477 \times 10^{-12}$ & $6.31398 \times 10^{-12}$ & $1.87240 \times 10^{-9}$ \\
2 & $4.88498 \times 10^{-15}$ & $1.84058 \times 10^{-12}$ & $1.36483 \times 10^{-11}$ & $2.72018 \times 10^{-9}$ \\
3 & $3.95517 \times 10^{-15}$ & $7.25758 \times 10^{-12}$ & $3.17973 \times 10^{-12}$ & $2.33563 \times 10^{-9}$ \\
4 & $9.21485 \times 10^{-15}$ & $3.07290 \times 10^{-11}$ & $2.27196 \times 10^{-11}$ & $9.2129 \times 10^{-9}$ \\
5 & $2.39808 \times 10^{-14}$ & $7.20091 \times 10^{-12}$ & $3.59939 \times 10^{-11}$ & $6.27719 \times 10^{-9}$ \\
6 & $1.31006 \times 10^{-14}$ & $5.29026 \times 10^{-11}$ & $1.25912 \times 10^{-11}$ & $7.89854 \times 10^{-9}$ \\
7 & $2.66454 \times 10^{-14}$ & $5.82896 \times 10^{-11}$ & $3.45277 \times 10^{-11}$ & $1.92327 \times 10^{-8}$ \\
8 & $5.81757 \times 10^{-14}$ & $1.54483 \times 10^{-11}$ & $5.94373 \times 10^{-11}$ & $1.06178 \times 10^{-8}$ \\
9 & $3.63043 \times 10^{-14}$ & $5.10261 \times 10^{-11}$ & $2.78653 \times 10^{-11}$ & $1.50077 \times 10^{-8}$ \\
10 & $4.32987 \times 10^{-14}$ & $9.03253 \times 10^{-11}$ & $4.08553 \times 10^{-11}$ & $3.24998 \times 10^{-8}$
\end{tabular}

Table 19: Comparison of absolute errors of MG4, MG6, RK4 and RK6 solutions for $x(t)$ for the time interval $(0,10)$ with the step size $h=1 / 100$ of equation (61).

\begin{tabular}{lllll}
\hline $\mathrm{t}$ & MG6 & RK6 & MG4 & RK4 \\
\hline 0 & 0 & $4.76456 \times 10^{-21}$ & 0 & $1.16996 \times 10^{-20}$ \\
1 & $2.66453 \times 10^{-15}$ & $6.40809 \times 10^{-12}$ & $4.56063 \times 10^{-12}$ & $4.44138 \times 10^{-9}$ \\
2 & $2.99066 \times 10^{-15}$ & $6.22117 \times 10^{-10}$ & $5.69621 \times 10^{-12}$ & $3.12623 \times 10^{-9}$ \\
3 & $7.21644 \times 10^{-15}$ & $1.64857 \times 10^{-10}$ & $2.22036 \times 10^{-11}$ & $7.73299 \times 10^{-9}$ \\
4 & $1.64313 \times 10^{-14}$ & $7.99509 \times 10^{-11}$ & $2.00843 \times 10^{-11}$ & $5.75861 \times 10^{-9}$ \\
5 & $3.92741 \times 10^{-15}$ & $1.06491 \times 10^{-9}$ & $1.00612 \times 10^{-11}$ & $1.01620 \times 10^{-8}$ \\
6 & $2.68673 \times 10^{-14}$ & $9.29750 \times 10^{-10}$ & $4.30804 \times 10^{-11}$ & $1.88528 \times 10^{-8}$ \\
7 & $4.30766 \times 10^{-14}$ & $7.62079 \times 10^{-12}$ & $4.00304 \times 10^{-11}$ & $9.55437 \times 10^{-10}$ \\
8 & $8.32667 \times 10^{-15}$ & $9.29978 \times 10^{-10}$ & $8.12926 \times 10^{-12}$ & $2.09217 \times 10^{-8}$ \\
9 & $5.79536 \times 10^{-14}$ & $1.40471 \times 10^{-10}$ & $6.13301 \times 10^{-11}$ & $2.67420 \times 10^{-8}$ \\
10 & $8.52651 \times 10^{-14}$ & $7.29638 \times 10^{-10}$ & $6.33613 \times 10^{-11}$ & $7.37761 \times 10^{-9}$
\end{tabular}

Table 20: Comparison of absolute errors of MG4, MG6, RK4 and RK6 solutions for $x^{\prime}(t)$ for the time interval $(0,10)$ with the step size $h=1 / 100$ of equation (61).

[2] Bialynicki-Birula I., Mielnik B. \& Plebanski J. 1969. Explicit solution of the continuous Baker-Campbell-Hausdorff problem and a new expression for the phase operator. Ann. Phys. 51: 187-200.

[3] Blanes S. et al. 2009. The Magnus expansion and some of its applications, Physics Reports 470: 151-238.

[4] Blanes S. et al. 2014. Time-averaging and exponential integrators for non-homogeneous linear IVPs and BVPs. Applied Numerical Mathematics 62: 875-894.

[5] Blanes S. et al. 1998. Magnus and Fer expansions for matrix differential equations: the convergence problem. J. Phys. A 31 : 259-268. 

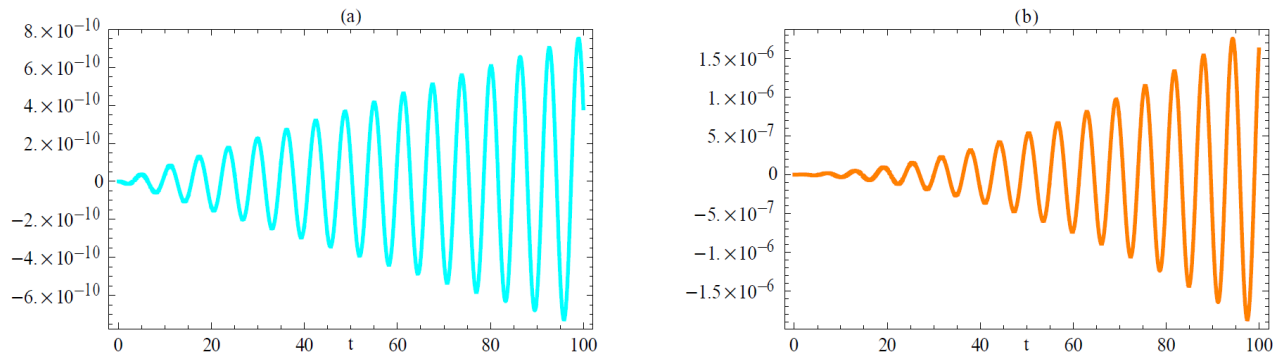

Fig. 10: Error graphics for $x(t)$ (a) MG4, (b) RK4 solution of equation (61).
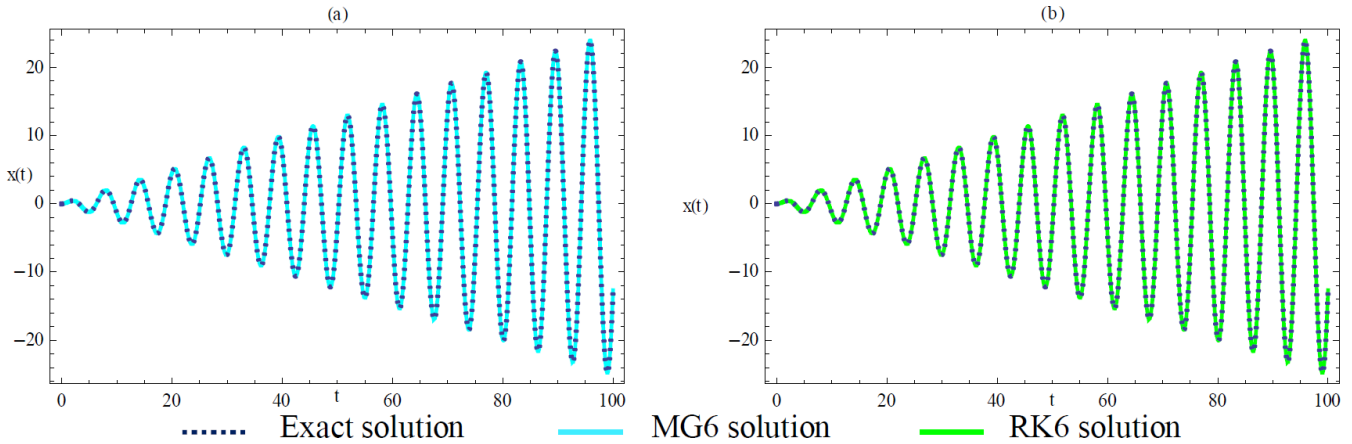

Fig. 11: Comparison of (a) MG4, (b) RK4 with exact solution for $x(t)$ in equation (61).
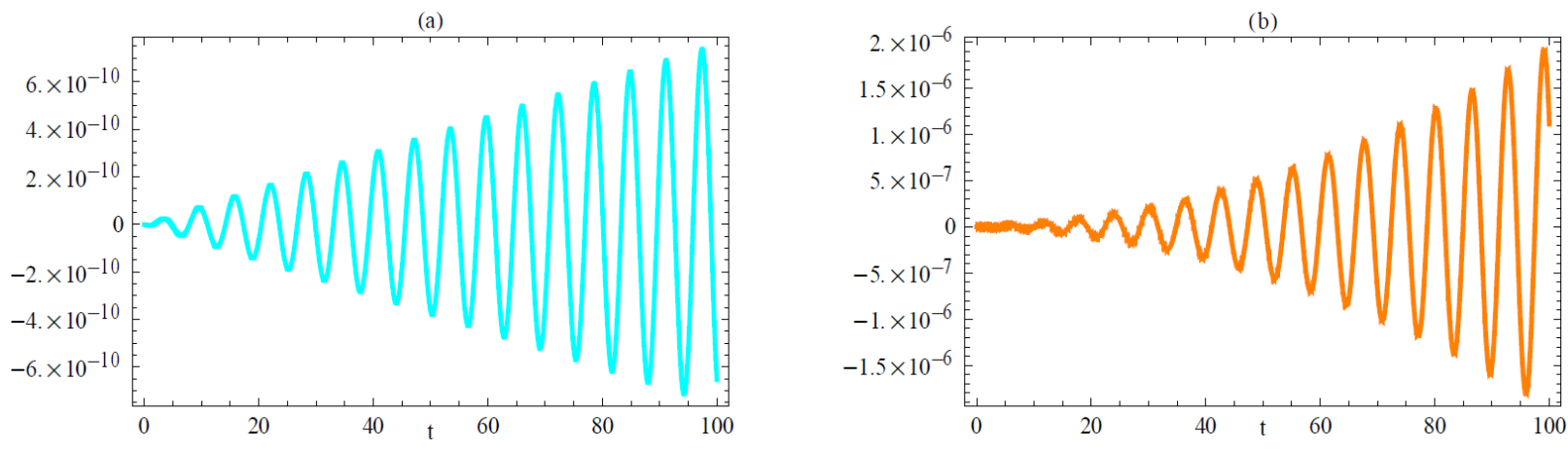

Fig. 12: Error graphics for $x^{\prime}(t)$ (a) MG4, (b) RK4 solution of equation (61).

[6] Boyce W.E., DiPrima R.C., 2001. "Elementary Differential Equations and Boundary Value Problems", John Wiley \& Sons Inc, Singapore, pp. 200-205.

[7] Cady W.A. 1974. Rotational spectral line broadening of OCS by noble gases. J. Chem. Phys. 60: 3318-23.

[8] Castellano A. et al. 2014. Geometric numerical integrators based on the Magnus expansion in bifurcation problems for non-linear elastic solids. Frattura ed Integrità Strutturale 29: 128-138.

[9] D’Olivo J.C. \& Oteo J.A. 1990. Magnus expansion and the two-neutrino oscillations in matter. Phys. Rev. D 42: 256-9.

[10] Dahmen H.D., Scholz B. \& Steiner F. 1982. Infrared dynamics of QED and the asymptotic behavior of the electron form factor. Nucl. Phys. B 202: 365-81.

[11] De Silva Clarence W. 2000. Vibration: Fundamentals and Practice. CRC Pres, Boca Raton. Pp. 25.

[12] Duleba I. 1997. Locally optimal motion planning of nonholonomic systems. Int. J. Robotic Systems 14: 767-788. 

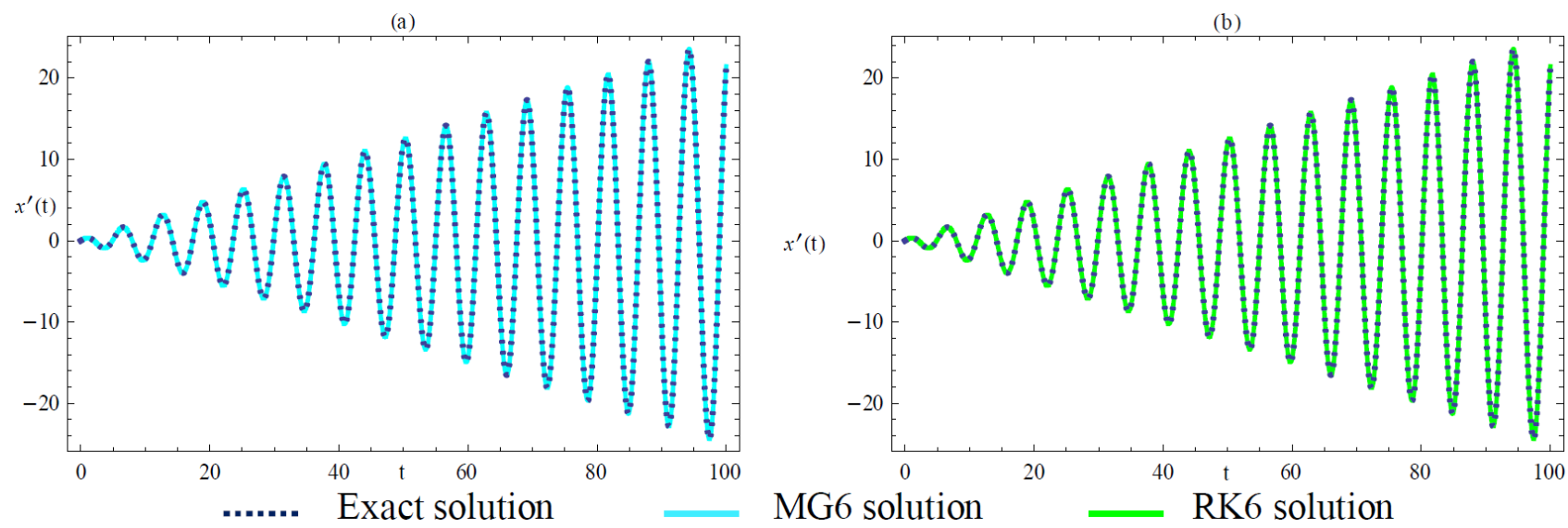

Fig. 13: Comparison of (a) MG4, (b) RK4 with exact solution for $x^{\prime}(t)$ in equation (61).
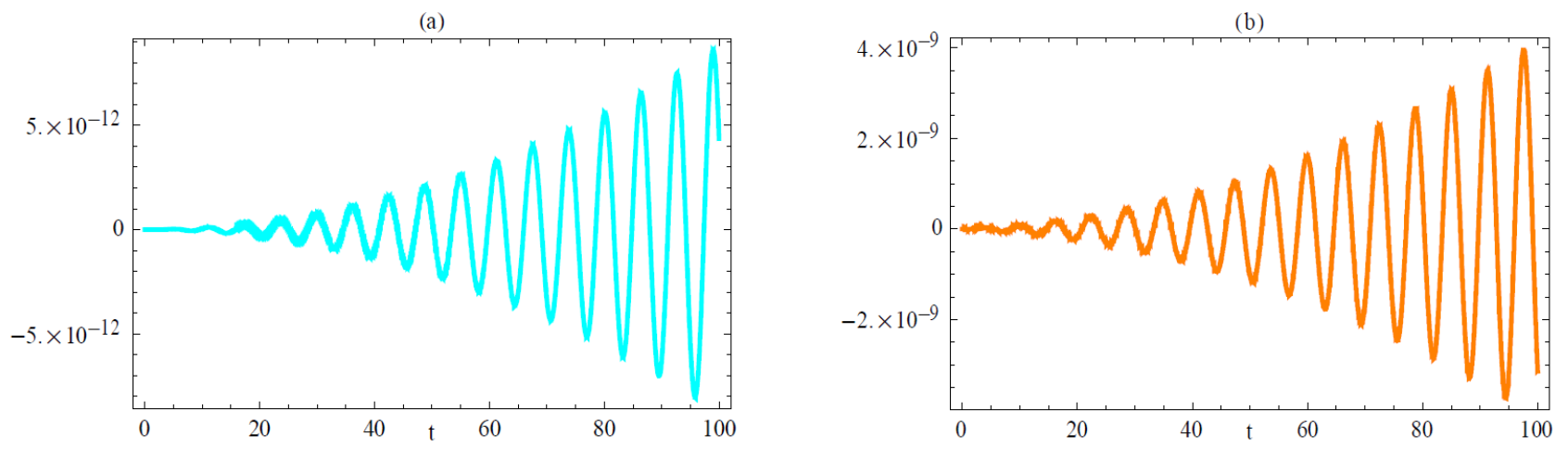

Fig. 14: Error graphics for $x(t)$ (a) MG6, (b) RK6 solution of equation (61).

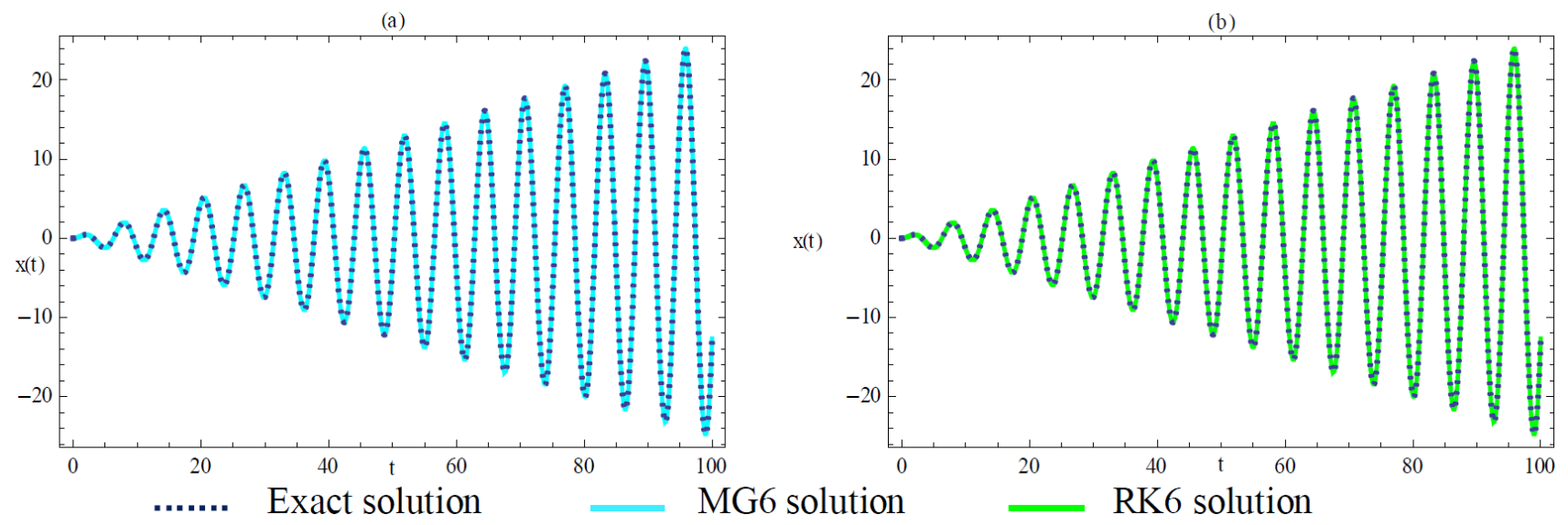

Fig. 15: Comparison of (a) MG6, (b) RK6 with exact solution for $x(t)$ in equation (61). 
(a)

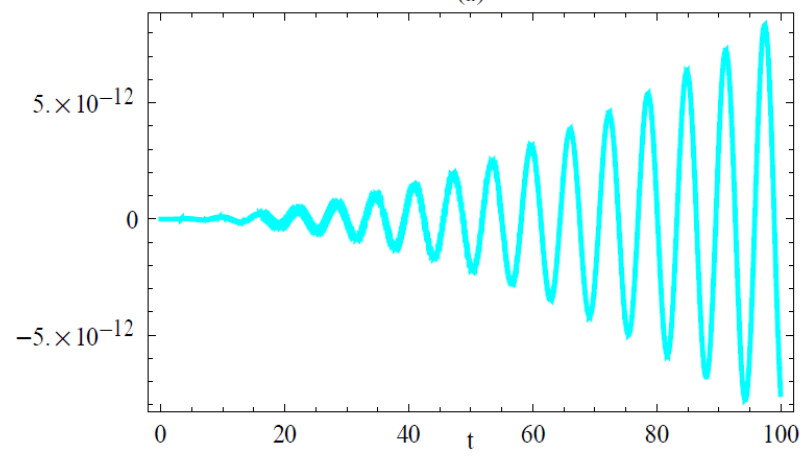

(b)

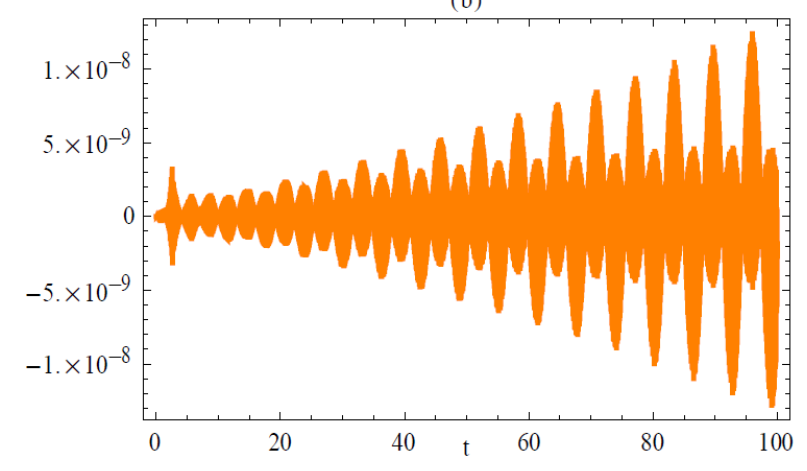

Fig. 16: Error graphics for $x^{\prime}(t)$ (a) MG6, (b) RK6 solution of equation (61).

(a)

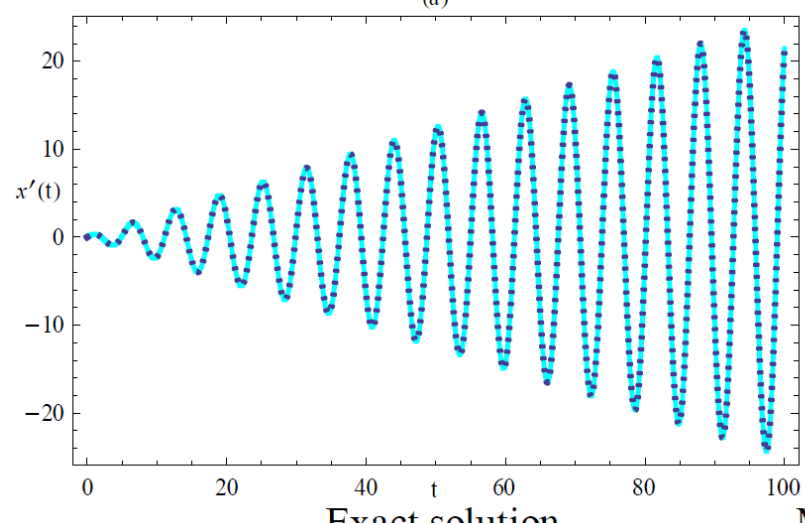

....... Exact solution $\quad$ MG6 solution

(b)

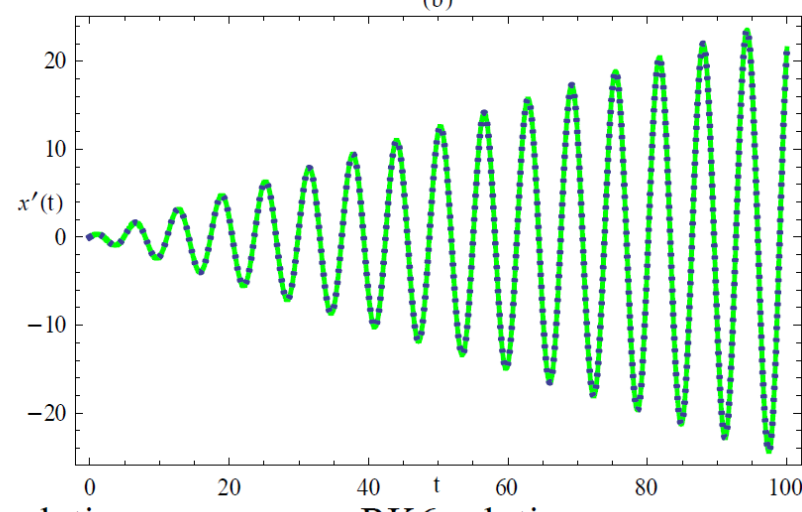

RK6 solution

Fig. 17: Comparison of (a) MG6, (b) RK6 with exact solution for $x^{\prime}(t)$ in equation (61).

[13] Duleba I. 1998. On a computationally simple form of the generalized Campbell-Baker-Hausdorff-Dynkin formula. Systems Control Letters 34: 191-202.

[14] Eichler J. 1977. Magnus approximation for K-shell ionization by heavy-ion impact. Phys. Rev. A 15: 1856-1862.

[15] Evans W.A.B. 1968. On some application of Magnus expansion in nuclear magnetic resonance. Ann. Phys. 48: $72-93$.

[16] Fomenko A.T. \& Chakon R.V. 1990. Recurrence formulas for homogeneous terms of a convergent series that represents a logarithm of a multiplicative integral on Lie groups. Funct. Anal. Appl. 1: 41-49.

[17] Hyman H.A. 1985. Dipole Magnus approximation for electron-atom collisions: Excitation of the resonance transitions of Li, Na and K. Phys. Rev. A 31: 2142-2148.

[18] Iserles A. \& Nørsett S.P. 1997. Linear ODEs in Lie groups. DAMTP tech report 1997/NA9 University of Cambridge.

[19] Iserles A. \& Nørsett S.P. 1999. On the solution of linear differential equations in Lie groups. Philosophical Transactions: Mathematical, Physical and Engineering Sciences 357: 983-1020.

[20] Iserles A. et al. 2000. Lie-group Methods. Acta Numerica 9: 215-365.

[21] Iserles A., Marthinsen A. \& Nørsett S.P. 1999. On the implementation of the method of Magnus series for linear differential equations. BIT Numerical Mathematics Vol 39: 281-304.

[22] Iserles A., Nørsett S.P. \& Rasmussen A.F. 2001. Time-symmetry and high-order Magnus methods, Applied Numerical Mathematics. Volume 39: 379-401.

[23] Lu Y.Y. 2005. A fourth-order Magnus scheme for Helmholtz equation. J. Comput. Appl. Math. 173: 247-258.

[24] Lu Y.Y. 2006. Some techniques for computing propagation in optical waveguides. Comm. Comp. Phys. 1: $1056-1075$. 
[25] Lu Y.Y. 2007. A fourth order derivative-free operator marching method for Helmholtz equation in waveguides. J. Comput. Math. 25: 719-729.

[26] Magnus W. 1954. On the exponential solution of differential equations for a linear operator. Comm. Pure and Appl. Math. 7: 639-673.

[27] Mielnik B. \& Plebanski J. 1970. Combinatorial approach to Baker-Campbell-Haussdorf exponents. Ann. Inst. Henri Poincare A 12: $215-254$.

[28] Milfeld K.F. \& Wyatt R.E. 1983. Study extension and application of Floquet theory for quantum molecular systems in an oscillating field. Phys. Rev. A 27: 72-94.

[29] Moan P.C. \& Niesen J. 2008. Convergence of the Magnus series. Found. Comput. Maths 8: 291-301.

[30] Murray R.M., Li Z. \& Satry S.S. 1994. A Mathematical Introduction to Robotic Manipulation. CRC Pres, London.

[31] Oteo J.A. \& Ros J. 1991. The Magnus expansion for classical Hamiltonian systems. J. Phys. A: Math. Gen. 24: 5751-62.

[32] Pechukas P. \& Light J.C. 1966. On the exponential form of time-displacement operators in quantum mechanics. J. Chem. Phys. 44: 3897-912.

[33] Robinson D.W. 1963. Multiple Coulomb excitations of deformed nuclei. Helv. Phys. Acta 36: 140-54.

[34] Ross S.L. 1984. Differential Equations, John Wiley \& Sons Inc, Singapore. Pp. 179-203.

[35] Schek I., Jortner J. \& Sage M.L. 1981. Application of the Magnus expansion for high-order multiphoton excitation. Chem. Phys. 59: 11-27.

[36] Strichartz R.S. 1987. The Campbell-Baker-Hausdorff-Dynkin formula and solutions of differential equations. J. Func. Anal. 72: $320-345$.

[37] Wille U. \& Hippler R. 1986. Mechanisms of inner-shell vacancy production in slow ion-atom collisions. Phys. Rep. 132: 129-260.

[38] Wille U. 1981. Magnus expansion for rotationally induced inner-shell excitation. Phys. Lett. A 82: 389-392. 